\title{
THE STEFAN PROBLEM IN SEVERAL SPACE VARIABLES
}

\author{
BY \\ AVNER FRIEDMAN( $\left.{ }^{1}\right)$
}

Introduction. The Stefan problem is a free boundary problem for parabolic equations. The solution is required to satisfy the usual initial-boundary conditions, but a part of the boundary is free. Naturally, an additional condition is imposed at the free boundary. A two-phase problem is such that on both sides of the free boundary there are given parabolic equations and initial-boundary conditions, and neither of the solutions is identically constant. In case the space-dimension is one, there are numerous results concerning existence, uniqueness, stability, and asymptotic behavior of the solution; we refer to [1] and the literature quoted there (see also [8]).

In the case of several space variables the problem is much harder. The difficulty is not merely due to mathematical shortcomings but also to complications in the physical situation. Thus, even if the data are very smooth the solution need not be smooth, in general. For example, when a body of ice having the shape

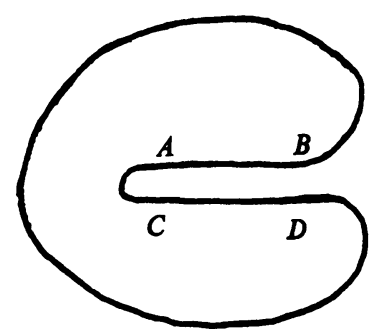

keeps growing, the interfaces $A B$ and $C D$ may eventually coincide. Then, in the next moment the whole joint boundary will disappear. Thus the free boundary varies in a discontinuous manner.

This example motivates one to look for "weak" solutions. In [4] the concept of a weak solution is defined. Furthermore, existence and uniqueness theorems are proved. The existence proofs are based on a finite-difference approximation.

In the present work we give a simpler derivation of the existence theorems of [4]. Our method has also the advantages that (i) it yields better inequalities on the solution and on its first derivatives than in [4], and (ii) it enables us to find certain

Received by the editors January 27, 1967 and, in revised form, April 6, 1967.

( ${ }^{1}$ ) This work was supported by the Guggenheim Fellowship and by the National Science Foundation NSF Grant GP-5558. 
regions where the weak solution is a smooth function. We also derive (in \$4) a stability theorem for the weak solution. This roughly states that, in the $L^{2}$ norm, the solution varies continuously with the initial and boundary data. In $\S 5$ we prove an asymptotic convergence (in $L^{2}$ ) of the solution as $t \rightarrow \infty$. Some generalizations of the results of $\S \S 2-5$ are given in $\S 6$.

In the next sections (except for the last one) we are concerned with the one-phase problem. Existence and uniqueness of weak solutions follow by the methods for the two-phase problem. We also derive asymptotic bounds on the set where the solution is (i) positive and smooth, (ii) identically zero. Asymptotic bounds for classical solutions of the Stefan problem, in several space variables, were obtained by a different method in [2].

In the definition of a weak solution enters a function $a(u)$ defined by

$$
\begin{aligned}
& a(u)=\alpha_{1} u \quad \text { if } u>0 \text {, } \\
& =\alpha_{2} u-\alpha \text { if } u \leqq 0,
\end{aligned}
$$

where $\alpha_{1}, \alpha_{2}, \alpha$ are positive constants which occur in the setting of the problem. For each weak solution $u(x, t)$ ( $u$ is a bounded measurable function) the function $a(u(x, t))$ is defined as $\alpha_{1} u(x, t)$ if $u(x, t)>0$ and as $\alpha_{2} u(x, t)-\alpha$ if $u(x, t)<0$; at the points where $u(x, t)=0$ the function $a(u(x, t))$ is only required to satisfy $-\alpha \leqq$ $a(u(x, t)) \leqq 0$ and to be such that it is altogether a measurable function. The set where $a(u(x, t)) \geqq 0$ can be interpreted as being the set occupied by the liquid of the problem, whereas the set where $a(u(x, t)) \leqq-\alpha$ can be considered to be the set occupied by the solid of the problem. It is of fundamental interest to find the nature of the set $W$ where $-\alpha<a(u(x, t))<0$. This set may be conceived as a "cloud" lying between the solid and the liquid.

In $\$ 8$ we show (for the one-phase problem) that the set $G_{1}(\sigma)$ occupied by the liquid at time $t=\sigma$ increases with $\sigma$. Some results on the strict increase of $G_{1}(\sigma)$ are given in $\S 9$. The result of $\S 8$ is used in $\S 10$ to prove that the set $W(\sigma) \equiv W$ $\cap\{t=\sigma\}$ is contained in the boundary of $G_{1}(\sigma)$. Thus, in particular, $W$ has no interior points. Miscellaneous results on the one-phase problem are given in $\S 11$. Finally, in $\$ 12$ we consider briefly other free boundary problems.

All the functions in this paper are real valued.

1. Reduction of the classical problem to a generalized one. Let $G$ be a bounded domain in $R^{n}$, whose boundary consists of two $C^{1}$ hypersurfaces: $\partial_{1} G$ and $\partial_{2} G$, and let $\partial_{1} G$ lie in the interior of $\partial_{2} G$. For any $T, 0<T \leqq \infty$, set $\Omega_{T}=G \times(0, T)$. We introduce the elliptic operators

$$
L_{i}=\sum_{j, k=1}^{n} a_{j k}^{i}(x, t) \frac{\partial^{2}}{\partial x_{j} \partial x_{k}}+\sum_{j=1}^{n} b_{j}^{i}(x, t) \frac{\partial}{\partial x_{j}}+c^{i}(x, t) \quad(i=1,2)
$$

with coefficients satisfying: $a_{j k}^{i}, \nabla_{x} a_{j k}^{i}, \nabla_{x}^{2} a_{j k}^{i}, b_{j}^{i}, \nabla_{x} b_{j}^{i}, c^{i}$ are continuous in $\bar{\Omega}_{\infty}$. 
Consider the following system of equations for a triple $\left(u_{1}, u_{2}, \Phi\right)$ :

$$
\begin{aligned}
\partial u_{i} / \partial t & =L_{i} u_{i} \text { for } x \in G(t), 0<t<T(i=1,2), \\
u_{i} & =g_{i} \text { for } x \in \partial_{i} G, 0<t<T(i=1,2),
\end{aligned}
$$

where $g_{1}>0, g_{2}<0$.

$$
u_{i}=h_{i} \text { on } G_{i}(0)(i=1,2)
$$

where $h_{1}>0, h_{2}<0$.

$$
\begin{gathered}
u_{i}=0 \text { for } x \in \Gamma(t), 0 \leqq t<T(i=1,2) . \\
\sum_{j, k=1}^{n} a_{j k}^{1} \frac{\partial u_{1}}{\partial x_{j}} \frac{\partial \Phi}{\partial x_{k}}-\sum_{j, k=1}^{n} a_{j k}^{2} \frac{\partial u_{2}}{\partial x_{j}} \frac{\partial \Phi}{\partial x_{k}}=\alpha \frac{\partial \Phi}{\partial t} \quad \text { for } x \in \Gamma(t), 0<t<T,
\end{gathered}
$$

where $\alpha$ is a positive constant. Here $\Gamma(t)$ is a hypersurface lying in $G(t)=G \times\{t\}$, $G_{i}(t)$ in the domain lying in $G(t)$ and bounded by $\Gamma(t)$ and $\partial_{i} G(t)=\partial_{i} G \times\{t\}$, and $\Phi(x, t)$ is a $C^{1}$ function in $\bar{\Omega}_{T}$ such that $\Gamma(t)=\left\{(x, t) \in \bar{\Omega}_{T} ; \Phi(x, t)=0\right\}, \nabla_{x} \Phi(x, t) \neq 0$ on $\Gamma(t), \Phi(x, t)<0$ in $G_{1}(t)$, and $\Phi(x, t)>0$ in $G_{2}(t)$. The functions $h_{i}, g_{i}$ are the initial and boundary data for $u_{i}$, and $S=\bigcup_{0 \leqq t<T} \Gamma(t)$ is the "free boundary".

The classical (two-phase) Stefan problem is to find a solution $\left(u_{1}, u_{2}, \Phi\right)$ of (1.1)-(1.5).

We recall that the transversal vector $\mu_{i}$ with respect to $L_{i}$ at $\Gamma(t)$ is given by $\left\{\sum a_{j k}^{i} \cos \left(x_{k}, \nu\right)\right\}$ where $\nu$ is the normal vector on $\Gamma(t)$. We shall always take the normal $\nu$ on $\Gamma(t)$ to point into $G_{2}(t)$. Since $\partial \Phi / \partial x_{k}=\lambda \cos \left(x_{k}, v\right), \partial \Phi / \partial t=\lambda \cos (t, v)$ for some $\lambda \neq 0$, we can write (1.5) in the form

$$
\partial u_{1} / \partial \mu_{1}-\partial u_{2} / \partial \mu_{2}=\alpha \Phi_{t} /|\nabla \Phi| \quad\left(|\nabla \Phi|^{2}=\Phi_{t}^{2}+\left|\nabla_{x} \Phi\right|^{2}\right) .
$$

It has already been pointed out in the Introduction that classical solutions do not exist in general (even for smooth coefficients, data and $\partial_{1} G, \partial_{2} G$ ). We shall therefore transform the problem (1.1)-(1.5) into a "generalized" one, as in [4].

Set $\partial G=\partial_{1} G \cup \partial_{2} G$,

$$
\begin{aligned}
a(u) & =u-\alpha \quad \text { if } u \leqq 0, \\
& =u \quad \text { if } u>0, \\
g & =g_{1} \quad \text { on } \partial_{1} G(t), \\
& =g_{2} \quad \text { on } \partial_{2} G(t), \\
h & =h_{1} \quad \text { on } G_{1}(0), \\
& =h_{2} \quad \text { on } G_{2}(0), \\
a_{i j}(x, t, u) & =a_{i j}^{1}(x, t) \quad \text { for }(x, t) \in \bar{\Omega}_{\infty}, u \geqq 0, \\
& =a_{i j}^{2}(x, t) \quad \text { for }(x, t) \in \bar{\Omega}_{\infty}, u<0 .
\end{aligned}
$$

We define $b_{i}(x, t, u), c(x, t, u)$ in a similar way to $a_{i j}(x, t, u)$ and then set

$$
L u=\sum_{j, k=1}^{n} a_{j k}(x, t, u) \frac{\partial^{2} u}{\partial x_{j} \partial x_{k}}+\sum_{j=1}^{n} b_{j}(x, t, u) \frac{\partial u}{\partial x_{j}}+c(x, t, u) u .
$$


Denote by $L_{i}^{*}$ the adjoint of $L_{i}$, and define $L^{*} u$ by $L^{*} u=L_{1} u$ at points where $u>0$ and $L^{*} u=L_{2} u$ at points where $u .<0$. The function $\mu_{i}$ on $\partial_{i} G(t)$ is defined as the outward transversal vector with respect to $L_{i}$. Set

$$
\begin{aligned}
& \mu=\mu_{1} \text { on } \partial_{1} G(t), \\
& =\mu_{2} \text { on } \partial_{2} G(t) \text {. }
\end{aligned}
$$

DEFinition. A bounded measurable function $u$ in $\Omega_{T}$ is called a weak (or generalized) solution of (1.1)-(1.5) if

$$
\iint_{\Omega_{T}}\left[u L^{*} \phi+a(u) \frac{\partial \phi}{\partial t}\right] d x d t=\int_{0}^{T} \int_{\partial G} g \frac{\partial \phi}{\partial \mu} d S_{x} d t-\int_{G(0)} a(h) \phi d x
$$

holds for any function $\phi$ in $\bar{\Omega}_{T}$ with $\nabla_{x} \phi, \nabla_{x}^{2} \phi, D_{t} \phi$ continuous in $\bar{\Omega}_{T}$ and $\phi=0$ on $G(T)$ and on $\partial G \times(0, T)$.

In this definition, $a(u(x, t))$ is not to be defined by (1.7) when $u(x, t)=0$. Instead, it is defined as any function $\beta(x, t)$ subject to the following restrictions: (i) $a(u(x, t))$ is a measurable function in $\Omega_{T}$, and (ii) $a(0-0)=-\alpha \leqq \beta(x, t) \leqq 0=a(0+0)$. The functions $a_{j k}(x, t, u(x, t)), b_{j}(x, t, u(x, t)), c(x, t, u(x, t))$ are defined in a similar way when $u(x, t)=0$. Since, however, on the set where $u=0$ the integrand $u L^{*} \phi$ is equal to zero, the latter definitions are of no significance.

By a classical solution of (1.1)-(1.5) we mean a solution $\left(u_{1}, u_{2}, \Phi\right)$ of (1.1)-(1.5) such that $u_{i}, \nabla_{x} u_{i}$ are continuous in $\bigcup_{0 \leqq t<T} \mathrm{Cl}\left(G_{i}(t)\right)$ (here $\mathrm{Cl}(A)$ stands for the closure of $A)$ and $\nabla_{x}^{2} u_{i}, D_{t} u_{i}$ are continuous in $\bigcup_{0<t<T} G_{i}(t)$.

THEOREM 1. A classical solution of (1.1)-(1.5) in $\Omega_{T}$ is also a weak solution of (1.1)-(1.5) in $\Omega_{T}$.

THEOREM 2. Let $u$ be a weak solution of (1.1)-(1.5) in $\Omega_{T}$. Assume that there exists a $C^{1}$ function $\Phi$ in $\bar{\Omega}_{T}$ satisfying

$\Gamma(t) \equiv\left\{(x, t) \in \Omega_{T} ; u(x, t)=0\right\}=\left\{(x, t) \in \Omega_{T} ; \Phi(x, t)=0\right\}$ and $\nabla_{x} \Phi \neq 0$ on $\Gamma(t)$, $\Phi<0$ in $G_{1}(t), \Phi>0$ in $G_{2}(t)$, where $G_{i}(t)$ is the domain bounded by $\Gamma(t)$ and $\partial_{i} G(t)$. Setting $u_{i}=u$ in $G_{i}(t)$, assume that $u_{i}, \nabla_{x} u_{i}$ are continuous in $\bigcup_{0 \leqq t<T} \mathrm{Cl}\left(G_{i}(t)\right)$ and that $\nabla_{x}^{2} u_{i}, D_{t} u$ are continuous in $\bigcup_{0<t<T} G_{i}(t)$. Then $\left(u_{1}, u_{2}, \Phi\right)$ is a classical solution of (1.1)-(1.5).

For the sake of completeness we give here the proofs (which are given also in [4], for the case $n=1$ ).

Proofs. To prove Theorem 1 we multiply both sides of (1.1) for $i=1$ by $\phi$ and integrate over $\cup G_{1}(t)$. Recalling that $u_{1}=0$ on $\Gamma(t)$, we obtain, after integration by parts,

$$
\begin{array}{r}
\int_{0}^{T} \int_{G_{1}(t)}\left(u_{1} \frac{\partial \phi}{\partial t}+u_{1} L_{1}^{*} \phi\right) d x d t+\int_{0}^{T} \int_{\Gamma(t)} \phi \sum_{j, k} a_{j k}^{1} \frac{\partial u_{1}}{\partial x_{k}} \cos \left(x_{j}, v\right) d S_{x} d t \\
=\int_{0}^{T} \int_{\partial G_{1}} u_{1} \frac{\partial \phi}{\partial \mu_{1}} d S_{x} d t-\int_{G_{1}(0)} h_{1} \phi d x
\end{array}
$$


Writing $\partial u_{2} / \partial t=\partial\left(u_{2}-\alpha\right) / \partial t$ in (1.1) for $i=2$, and multiplying by $\phi$, we obtain, upon integrating over $\bigcup G_{2}(t)$,

$$
\begin{array}{r}
\int_{0}^{T} \int_{\Gamma(t)}(-\alpha) \phi \cos (t, \nu) d S_{x} d t+\int_{0}^{T} \int_{G_{2}(t)}\left[\left(u_{2}-\alpha\right) \frac{\partial \phi}{\partial t}+u_{2} L_{2}^{*} \phi\right] d x d t \\
-\int_{0}^{T} \int_{\Gamma(t)} \phi \sum_{j, k} a_{j k}^{2} \frac{\partial u_{2}}{\partial x_{k}} \cos \left(x_{j}, \nu\right) d S_{x} d t \\
=\int_{0}^{T} \int_{\partial_{2} G} u_{2} \frac{\partial \phi}{\partial \mu_{2}} d S_{x} d t-\int_{G_{2}(0)}(h-\alpha) \phi d x
\end{array}
$$

Set $u=u_{1}$ for $x \in G_{1}(t), u=u_{2}$ for $x \in G_{2}(t)$. Adding (1.10), (1.11), and using (1.5), the equation (1.9) follows.

Suppose, conversely, that $u$ is a weak solution satisfying the assumption of Theorem 2. Taking in (1.9) $\phi$ with compact support in $\bigcup G_{i}(t)$, we find that $u_{i}$ is a "weak" solution of (1.1). Since $u_{i}$ is smooth, it is easily seen to be a (classical) solution of (1.1). Next, taking $\phi$ in (1.9) with support in a neighborhood of a point lying on $\partial_{i} G(t)$ and integrating by parts, we find that (1.2) holds. (1.3) is proved in a similar way. Taking $\phi$ in (1.9) with support in a neighborhood of a point $(x, s)$, where $x \in \Gamma(s)$, and with $\phi=0$ on $\Gamma(t)$, and integrating by parts, we find that (1.4) holds. Finally, if we take in (1.9) $\phi$ with support in a neighborhood of a point $(x, s)$ where $x \in \Gamma(s)$ and use (1.4), we see that (1.5) is valid.

In the following sections we restrict ourselves, for simplicity, to elliptic operators $L_{i}$ with $a_{j k}^{i} \equiv A_{i} \delta_{j k}$ ( $A_{i}$ constant), $b_{j}^{i} \equiv 0$, but our methods extend also to the case where $a_{j k}^{i}=A_{i} a_{j k}, b_{j}^{i}=A_{i} b_{j}$ (see $\S 6$ ). We also assume that $c^{t}(x, t) \leqq 0$. It will be convenient to write the parabolic equations in the form

$$
\alpha_{i} \partial u_{i} / \partial t=\Delta u_{i}+c^{i}(x, t) u_{i}
$$

where $\alpha_{i}$ is a positive constant and $\Delta$ is the Laplace operator. We accordingly set

$$
L_{i}=\Delta+c^{i}
$$

We introduce

$$
\begin{aligned}
a(u) & =\alpha_{1} u \quad \text { if } u>0, \\
& =\alpha_{2} u-\alpha \quad \text { if } u \leqq 0, \\
g & =g_{i} / \alpha_{i} \quad \text { on } \partial_{i} G(t), \\
h & =h_{i} / \alpha_{i} \quad \text { on } G_{i}(0), \\
u & =u_{1} / \alpha_{1} \quad \text { if } u_{1}>0 \\
& =u_{2} / \alpha_{2} \quad \text { if } u_{2}<0
\end{aligned}
$$

Then (1.9) is still the equation for weak solutions of (1.1)-(1.5), with $L_{i}$ given by (1.13). Note that $\mu_{i}$ is now the normal $\nu$.

We conclude this section with the observation that if $u$ is a weak solution in $\Omega_{T}$, then it is also a weak solution in $\Omega_{\sigma}$ for any $\sigma<T$. Indeed, let $\chi_{\varepsilon}(t)$ be a $C^{\infty}$ function 
such that $\chi_{\varepsilon}(t)=1$ if $t<\sigma-\varepsilon, \chi_{\varepsilon}(t)=0$ if $t>\sigma$, and $\chi_{\varepsilon}^{\prime}(t) \leqq 0$. Let $\phi$ be as in (1.9) with $T$ replaced by $\sigma$. Apply (1.9) (in $\Omega_{T}$ ) with $\phi$ replaced by $\phi \chi_{\varepsilon}$ and take $\varepsilon \rightarrow 0$.

2. Existence and uniqueness of weak solutions. Unless the contrary is explicitly stated, we shall always assume that $T<\infty$.

The following assumptions will be needed:

(i) $\partial G$ is of class $C^{2+\eta}$ for some $\eta>0$.

(ii) $c^{i}(x, t) \equiv c^{i}(x)$ (for $\left.i=1,2\right)$ are Hölder continuous in $\bar{G}$, and $c^{i}(x) \leqq 0$.

(iii) $g_{1} \geqq \gamma_{1}>0$ on $\partial_{1} G \times[0, T]$ and $g_{2} \leqq \gamma_{2}<0$ on $\partial_{2} G \times[0, T]$, where $\gamma_{1}, \gamma_{2}$ are constants.

(iv) $h_{1} \geqq 0$ in $\mathrm{Cl}\left(G_{1}(0)\right)$ and $h_{1}=0$ only on $\Gamma(0) ; h_{2} \leqq 0$ on $\mathrm{Cl}\left(G_{2}(0)\right)$ and $h_{2}=0$ only on $\Gamma(0)$.

(v) $g, h$ are continuous functions in $\partial G \times[0, T]$ and $\mathrm{Cl}(G(0))$ respectively, and $g=h$ on $\partial G$.

Sometimes we shall need stronger assumptions on $g, h$; namely:

(vi) $h$ is continuous in $\mathrm{Cl}(G(0))$ and it belongs to $W^{1,2}(G)$ (i.e. it has first strong derivatives in $L^{2}(G)$ ).

(vii) There exists a function $\Psi$ with $\nabla_{x} \Psi, \nabla_{x}^{2} \Psi, D_{t} \Psi$ Hölder continuous (say, exponent $\eta$ ) in $\bar{\Omega}_{T}$, such that $\Psi=g$ on $\partial G \times[0, T]$ and $\Psi=h$ in $V \cap G(0)$, where $V$ is an $(n+1)$-dimensional neighborhood of $\partial G$.

REMARK. (vii) is equivalent to stating that (a) $g$ has two continuous derivatives with respect to $x \in \partial G$ and one continuous derivative with respect to $t,(\mathrm{~b}) h(x)$ is in $C^{2+\eta}$ in some neighborhood of $\partial G$, and (c) $g, h$ satisfy some consistency conditions on $\partial G(0)$.

THEOREM 3. Assume that (i)-(iv), (vi), (vii) hold. Then there exists a weak solution $u$ of (1.1)-(1.5). $u$ belongs to $W^{1,2}\left(\Omega_{T}\right)$ and moreover, the function $\int_{G}\left|\nabla_{x} u(x, t)\right|^{2} d x$ is essentially bounded.

In [4] the assertion that $u \in W^{1,2}\left(\Omega_{T}\right)$ was proved only under the restrictions that $g$ is independent of $t$ and $c^{i} \equiv 0$. The last assertion of Theorem 3 was not proved in [4].

Proof of Theorem 3. Let $\left\{a_{m}(u)\right\}$ be a sequence of smooth functions such that $a_{m}(u) \rightarrow a(u)$ uniformly in any closed interval which excludes the origin. We may choose the $a_{m}(u)$ in such a way that

$$
\alpha_{3} \leqq a_{m}^{\prime}(u) \quad \text { where } \alpha_{3}=\min \left(\alpha_{1}, \alpha_{2}\right) \text {. }
$$

Let $\left\{\gamma_{m}(u)\right\}$ be a sequence of smooth functions such that $\gamma_{m}(u) \rightarrow \operatorname{sgn} u$ uniformly in any closed interval which excludes the origin. We may choose the $\gamma_{m}(u)$ so that

$$
-1 \leqq \gamma_{m}(u) \leqq 1
$$

Define

$$
\begin{aligned}
c_{m}(x, u) & =\frac{1}{2} c^{1}(x)\left[1+\gamma_{m}(u)\right]+\frac{1}{2} c^{2}(x)\left[1-\gamma_{m}(u)\right], \\
L^{m} u & =\Delta u+c_{m}(x, u) u
\end{aligned}
$$


Consider the system

$$
\begin{aligned}
a_{m}^{\prime}(v) \partial v / \partial t & =L^{m} v & & \text { in } \Omega_{T}, \\
v & =g & & \text { on } \partial G \times(0, T), \\
v & =h & & \text { on } G(0) .
\end{aligned}
$$

By [6] there exists a unique solution $v=v_{m}$. (One needs actually to assume that $h$ belongs to $C^{2+\eta}(G)$. But by standard approximation arguments (employing $a$ priori inequalities and barriers [1]) we find that a solution exists even if $h$ belongs to $C^{0}(G)$.)

We claim that there exists a $\delta_{0}$-neighborhood $W_{\delta_{0}}^{i}$ of $\partial_{i} G(i=1,2)$, independent of $m$, and an $\varepsilon_{0}>0$, also independent of $m$, such that

$$
(-1)^{i-1} v_{m}(x, t)>\varepsilon_{0} \quad \text { in } \Omega_{i}^{*}=\left(W_{\delta_{0}}^{i} \cap G\right) \times(0, T) .
$$

To prove this we consider the system

$$
\begin{array}{rlr}
L^{m} w & =0 & \\
w & =\varepsilon & \text { in } G, \\
w & =-K & \text { on } \partial_{1} G, \\
& \text { on } \partial_{2} G
\end{array}
$$

where $K, \varepsilon$ are positive numbers, and $K>\sup \left|g_{2}\right|, \varepsilon<\inf g_{1}$. By a standard fixedpoint-theorem technique (using Schauder's estimates) we find that there exists a solution $w_{\varepsilon}$ of (2.9) (not necessarily unique). Furthermore, $w_{\varepsilon_{j}} \rightarrow w_{0}$ uniformly in $G$ as $j \rightarrow \infty$, where $\left\{\varepsilon_{j}\right\}$ is a sequence which decreases to zero and where $w_{0}$ is a solution of (2.9) when $\varepsilon=0$. By the maximum principle, $w_{0}<0$ in $G$; hence $w_{\varepsilon,}<h_{1}$ on $G_{1}(0)$ provided $j$ is sufficiently large. The inequality $w_{\varepsilon}<g$ on $\partial G \times(0, T)$ is also obvious. We shall now prove that $w_{\varepsilon_{j}}<h_{2}$ on $\mathrm{Cl}\left(G_{2}(0)\right)$ provided $j$ is sufficiently large. It suffices to show that $w_{0}<h_{2}$ on $\mathrm{Cl}\left(G_{2}(0)\right)$. We compare $w_{0}$ with the function $K v_{0}$, where $v_{0}$ satisfies

$$
\begin{aligned}
\Delta v_{0}-\gamma v_{0} & =0 & & \text { in } G, \\
v_{0} & =0 & & \text { on } \partial_{1} G, \\
v_{0} & =-1 & & \text { on } \partial_{2} G,
\end{aligned}
$$

and $\left|c_{m}\left(x, w_{0}\right)\right| \leqq \gamma$. Since $v_{0}<0$ in $G, \Delta v_{0}+c_{m}\left(x, w_{0}\right) v_{0}=\left[\gamma+c_{m}\left(x, w_{0}\right)\right] v_{0} \leqq 0$. Hence, by the maximum principle, $K v_{0}-w_{0} \geqq 0$ in $G$. Now, $v_{0}<0$ in $G$. Consequently, $K v_{0}<h_{2}$ on $\mathrm{Cl}\left(G_{2}(0)\right)$ if $K$ is sufficiently large. It follows that $w_{0}<h_{2}$ on $\mathrm{Cl}\left(G_{2}(0)\right)$.

Having proved that $v_{m}>w_{\varepsilon}$ on $G(0)$ and on $\partial G \times(0, T)$, we can apply the maximum principle and conclude that $v_{m}>w_{\varepsilon_{j}}$ in $\Omega_{T}$. This implies (2.8) for $i=1$. Since $c_{m}$ is bounded by a constant independent of $m$, by examining the above arguments we find that the constants $\varepsilon_{0}, \delta_{0}$ can be taken to be independent of $m$. The proof of $(2.8)$ for $i=2$ is similar. 
From (2.8) it follows that there exists an $m_{0}=m_{0}\left(\varepsilon_{0}, \delta_{0}\right)$ such that if $m \geqq m_{0}$ then $a_{m}^{\prime}\left(v_{m}\right)=\alpha_{i}, c_{m}(x, u)=c^{i}(x)$ provided $(x, t) \in \Omega_{i}^{*}$.

Consider $v_{m}$ in $\Omega_{i}^{*}$. It satisfies

$$
\begin{aligned}
\alpha_{i} \partial v_{m} / \partial t & =L_{i} v_{m} & & \text { in } \Omega_{i}^{*}, \\
v_{m} & =g & & \text { on } \partial_{i} G \times(0, T), \\
v_{m} & =h & & \text { on } \overline{\Omega_{i}^{*}} \cap\{t=0\} .
\end{aligned}
$$

Note also, by the maximum principle, that

$$
\left|v_{m}\right| \leqq A^{\prime} \quad \text { in } \Omega_{T}
$$

where $A^{\prime}=\max \{\sup |h|, \sup |g|\}$.

We shall prove

LEMMA 1. There is a constant $A^{\prime \prime}$ independent of $m$ such that

$$
\left|\partial v_{m} / \partial \nu\right| \leqq A^{\prime \prime} \quad \text { on } \partial G \times(0, T) \text {. }
$$

Proof. It suffices to prove $(2.12)$ on $\partial_{1} G \times(0, T)$. Introduce the function $w=v_{m}-\Psi$. It satisfies:

$$
\begin{aligned}
\alpha_{1} \partial w / \partial t & =L_{1} w+f & & \text { in } \Omega_{1}^{*}, \\
w & =0 & & \text { on } \partial_{1} G \times(0, T) \text { and on } \overline{\Omega_{1}^{*}} \cap\{t=0\},
\end{aligned}
$$

where $f \equiv L_{1} \Psi-\alpha_{1} \partial \Psi / \partial t$ is a bounded function. Let $(y, s)$ be any point on $\partial_{1} G \times(0, T)$ and denote by $\left(y_{0}, s\right)$ the center of a ball with radius $R=\left|y-y_{0}\right|$ which does not intersect $G(s)$. Then (compare [1, p. 87]) the function $w_{0}(x)$ $=k\left\{R^{-p}-\left|x-y_{0}\right|^{-p}\right\}$ for some positive $k, p$ satisfies

$$
\begin{aligned}
\alpha_{1} \partial w_{0} / \partial t & \geqq L_{1} w_{0}+1, \\
w_{0} & \geqq 0 \text { on } \partial_{1} G \times(0, T) \text { and on } \overline{\Omega_{1}^{*}} \cap\{t=0\}, \\
w_{0} & \geqq 1 \quad \text { on the remaining part of the boundary of } \Omega_{1}^{*} \text { which lies in } t<T .
\end{aligned}
$$

Let $k_{0}=\max \left\{\sup _{\Omega_{i}}|f|, \sup _{\Omega_{T}}|w|\right\}$. Applying the maximum principle to $k_{0} w_{0} \pm w$ we conclude that $k_{0} w_{0} \pm w \geqq 0$ in $\Omega_{1}^{*}$. Since $k_{0} w_{0} \pm w=0$ at $(y, s)$, we have $\partial\left(k_{0} w_{0} \pm w\right) / \partial \nu \leqq 0$ at $(y, s)$. Hence, $|\partial w / \partial \nu| \leqq-k_{0} \partial w_{0} / \partial \nu \leqq A^{*}$. Note that $k_{0}$ and, consequently, also $A^{*}$ are independent of $m$. (2.12) now follows.

We return to the system (2.5)-(2.7). We shall first suppose that $h$ is sufficiently smooth on $G$. Then $v_{m}$ is continuously differentiable in $\bar{G} \times[0, T]$. Multiplying both sides of (2.5) by $\partial v_{m} / \partial t$ and integrating over $\Omega_{\sigma}$, we obtain

$$
\begin{aligned}
\iint_{\Omega_{\sigma}} a_{m}^{\prime}\left(v_{m}\right)\left(\frac{\partial v_{m}}{\partial t}\right)^{2} d x d t+\iint_{\Omega_{\sigma}} \nabla v_{m} \cdot \nabla \frac{\partial v_{m}}{\partial t} d x d t \\
\quad=\int_{0}^{\sigma} \int_{\partial G} \frac{\partial v_{m}}{\partial \nu} \frac{\partial v_{m}}{\partial t} d S_{x} d t-\iint_{\Omega_{\sigma}} c_{m} v_{m} \frac{\partial v_{m}}{\partial t} d x d t
\end{aligned}
$$


Using (2.1)-(2.3), we get

$$
\begin{aligned}
\alpha_{3} \iint_{\Omega_{\sigma}}\left(\frac{\partial v_{m}}{\partial t}\right)^{2} d x d t & +\frac{1}{2} \int_{G(\sigma)}\left|\nabla v_{m}\right|^{2} d x \leqq \frac{1}{2} \int_{G(0)}|\nabla h|^{2} d x \\
& +\int_{0}^{\sigma} \int_{\partial G}\left|\frac{\partial v_{m}}{\partial \nu}\right|\left|\frac{\partial g}{\partial t}\right| d S_{x} d t+A \iint_{\Omega_{\sigma}}\left(v_{m}\right)^{2} d x d t,
\end{aligned}
$$

where $A$ is a constant independent of $m$.

Using Schwarz's inequality and (2.11), (2.12), it follows that

$$
\iint_{\Omega_{\sigma}}\left(\frac{\partial v_{m}}{\partial t}\right)^{2} d x d t+\int_{G(\sigma)}\left|\nabla v_{m}\right|^{2} d x \leqq A^{\prime} \int_{G(0)}|\nabla h|^{2} d x+A^{\prime}
$$

where $A^{\prime}$ is a constant independent of $m$.

If $h$ is not sufficiently smooth (but still satisfies (vi), (vii)), then we first take a sequence $\left\{h^{j}\right\}$ of smooth functions which converge to $h$ in the norm of $W^{1,2}(G)$ and which coincide with $h$ near $\partial G$. We apply (2.14) to each solution $v_{m}$ of (2.5)-(2.7) with $h$ replaced by $h^{j}$. We then take $j \rightarrow \infty$.

From (2.11), (2.14) it follows that there exists a subsequence of $\left\{v_{m}\right\}$, which we denote again by $\left\{v_{m}\right\}$, such that

$$
\begin{array}{cc}
v_{m} \rightarrow u & \text { in } L^{2}\left(\Omega_{T}\right), \\
\nabla_{x} v_{m} \rightarrow \nabla_{x} u & \text { in } L^{2}\left(\Omega_{T}\right), \\
D_{t} v_{m} \rightarrow D_{t} u & \text { in } L^{2}\left(\Omega_{T}\right),
\end{array}
$$

where " $\rightarrow$ " means weak convergence. Since bounded sets in $W^{1,2}\left(\Omega_{T}\right)$ are contained in compact subsets of $L^{2}\left(\Omega_{T}\right)$ (see [9]), we may also assume that

$$
\left\|v_{m}-u\right\|_{L^{2}\left(\Omega_{T}\right)} \rightarrow 0 \text {. }
$$

We may further assume that $\left\{v_{m}\right\}$ is almost everywhere convergent to $u$. Hence, by (2.11),

$$
|u(x, t)| \leqq \max \{\sup |h|, \sup |g|\} .
$$

From (2.15), (2.16), (2.18), and (2.14), it follows, by standard arguments, that $u$ belongs to $W^{1,2}\left(\Omega_{T}\right)$ and the function $\int|\nabla u(x, t)|^{2} d x$ is essentially bounded.

To complete the proof of the theorem, we shall show that $u$ is a weak solution.

Since $v_{m} \rightarrow u$ almost everywhere in $\Omega_{T}$, for almost all $(x, t)$ for which $u(x, t) \neq 0$ we have $a_{m}\left(v_{m}(x, t)\right) \rightarrow a(u(x, t))$. Next, the sequence $\left\{a_{m}\left(v_{m}(x, t)\right)\right\}$ is a bounded sequence of measurable functions. We may therefore assume that it converges weakly to some bounded measurable function $\beta(x, t)$. It then converges to $\beta$ also in measure, and we may assume that the convergence is also almost everywhere (otherwise we take a subsequence). It is now clear that $\beta(x, t)=a(u(x, t))$ for almost all the points $(x, t)$ for which $u(x, t) \neq 0$. If, on the other hand, $u(x, t)=0$, then, since $v_{m}(x, t) \rightarrow 0$, we conclude that $a(0-0) \leqq \beta(x, t) \leqq a(0+0)$. 
Next we may assume that the sequence $\left\{c_{m}\left(x, v_{m}(x, t)\right)\right\}$ is weakly convergent to some bounded measurable function $\delta(x, t)$. Hence it is also convergent in measure to $\delta(x, t)$. It follows that there exists a subsequence, which we again denote by $\left\{c_{m}\left(x, v_{m}(x, t)\right)\right\}$, such that it converges almost everywhere to $\delta(x, t)$. As easily seen from (2.3), $\delta(x, t)=c(x, u(x, t))$ for almost all $(x, t)$ for which $u(x, t) \neq 0$.

We shall now show that $u(x, t)$ satisfies (1.9). Multiply both sides of (2.5) by $\phi$ and integrate over $\Omega_{T}$. Using (2.6), (2.7), we obtain

$$
\begin{aligned}
\iint_{\Omega_{T}} a_{m}\left(v_{m}\right) \frac{\partial \phi}{\partial t} d x d t+\iint_{\Omega_{T}} v_{m}[\Delta \phi & \left.+c_{m}\left(x, v_{m}\right) \phi\right] d x d t \\
& =\int_{0}^{T} \int_{\partial G} g \frac{\partial \phi}{\partial \nu} d S_{x} d t-\int_{G(0)} a(h) \phi d x .
\end{aligned}
$$

Taking $m \rightarrow \infty$ and using (2.15), (2.18), we obtain (1.9).

COROllary 1. For any $0<\sigma<T$ and for every $\phi$ as in (1.9),

$$
\int_{\sigma}^{T} \int_{G}\left[u L^{*} \phi+a(u) \frac{\partial \phi}{\partial t}\right] d x d t=\int_{\sigma}^{T} \int_{\partial G} g \frac{\partial \phi}{\partial \mu} d S_{x} d t-\int_{G(\sigma)} a(u) \phi d x .
$$

Furthermore,

$$
\int_{G}\left|\nabla_{x} u(x, \sigma)\right|^{2} d \sigma \leqq B<\infty
$$

for all $\sigma \in(0, T]$.

Proof. We claim that for almost all $t$ in $(0, T)$,

$$
\begin{aligned}
v_{m}(x, t) & \rightarrow u(x, t), \\
a_{m}\left(v_{m}(x, t)\right) & \rightarrow a(u(x, t))
\end{aligned}
$$

in $L^{2}(G)$. Indeed, since $v_{m} \rightarrow u$ in $L^{2}\left(\Omega_{T}\right)$, for any $\phi \in L^{2}(G)$

$$
\int_{G} v_{m}(x, t) \phi(x) d x \rightarrow \int_{G} u(x, t) \phi(x) d x .
$$

Hence, by taking a subsequence we may assume that the convergence is also almost everywhere. Now take $\phi$ to vary in a dense sequence $\left\{\phi_{j}\right\}$ of $L^{2}(G)$. It follows that

$$
\int_{G} v_{m}(x, t) \phi(x) d x \rightarrow \int_{G} u(x, t) \phi(x) d x \quad \text { as } m \rightarrow \infty,
$$

for each $\phi=\phi_{j}$ and $t \in Z$ where $[0, T]-Z$ is a set of measure zero.

Since the $v_{m}$ are uniformly bounded, we deduce that (2.25) holds for all $\phi \in L^{2}(G)$ and $t \in Z$. This completes the proof of (2.22). The proof of (2.23) is similar. In what follows we denote by $Z$ a subset of $[0, T]$ such that (2.22) and (2.23) hold for all $t \in Z$ and such that $[0, T]-Z$ has measure zero.

Now multiply both sides of (2.5) by $\phi$ ( $\phi$ as in (1.9)) and integrate over $G \times(\sigma, T)$ where $\sigma \in Z$. Using (2.23) we obtain (2.20). 
We shall next redefine $u$ and $a(u)$ on $t=\sigma_{*}$, for any $\sigma_{*} \notin Z$, in such a way that (2.20) holds for $\sigma=\sigma_{*}$. We can choose a sequence $\left\{\sigma_{m}\right\} \subset Z$ converging to $\sigma_{*}$ such that

$$
u\left(x, \sigma_{m}\right) \rightarrow \gamma_{0}(x), \quad a\left(u\left(x, \sigma_{m}\right)\right) \rightarrow \gamma_{1}(x)
$$

as $m \rightarrow \infty$, where $\gamma_{0}, \gamma_{1}$ are some bounded measurable functions. By taking a subsequence we may assume that

$$
u\left(x, \sigma_{m}\right) \rightarrow \gamma_{0}(x), \quad a\left(u\left(x, \sigma_{m}\right)\right) \rightarrow \gamma_{1}(x)
$$

almost everywhere. But then it is clear that we may well redefine $u$ and $a(u)$ on $t=\sigma_{*}$ by

$$
u\left(x, \sigma_{*}\right)=\gamma_{0}(x), \quad a\left(u\left(x, \sigma_{*}\right)\right)=\gamma_{1}(x) .
$$

Writing (2.20) for $\sigma=\sigma_{m}$ and letting $m \rightarrow \infty$, we find, upon using (2.26), (2.28), that (2.20) holds for $\sigma=\sigma_{*}$.

We may assume that (2.21) holds for all $\sigma \in Z$. Using (2.26) it then follows that (2.21) holds also for $\sigma=\sigma_{*}$ where $\sigma_{*}$ is any point in $(0, T]-Z$.

COROLlaRY 2. If $n=1$ then $u(x, t)$ is a continuous function in $\bar{\Omega}_{T}$.

Proof. From (2.21) and the Sobolev inequality it follows that $u(x, t)$ is continuous in $x$, uniformly with respect to $t$. It remains to prove that $u\left(x_{0}, t\right)$ is continuous in $t$ for any $x_{0} \in \bar{G}$. The proof is analogous to an argument used in [10]. Suppose the assertion is false. Then there exists a sequence $\left\{t_{m}\right\}$ in $(0, T)$ and an $\varepsilon>0$, such that $t_{m} \rightarrow \tau$ and $\left|u\left(x_{0}, t_{m}\right)-u\left(x_{0}, \tau\right)\right|>\varepsilon$ for all $m$. By the continuity of $u(x, t)$ in $x$ it follows that the inequalities persist in some neighborhood $\left\{x ;\left|x-x_{0}\right|<\delta\right\} \cap \bar{G}$. Hence,

$$
\left|a\left(u\left(x, t_{m}\right)\right)-a(u(x, \tau))\right|>\gamma \varepsilon
$$

for some $\gamma>0$ independent of $m, \varepsilon$.

Writing (2.20) for $\sigma=t_{m}$ and $\sigma=\tau$, and subtracting one equation from another, we easily find that

$$
\int_{G}\left[a\left(u\left(x, t_{m}\right)\right)-a(u(x, \tau))\right] \phi(x, \tau) d x \rightarrow 0 \quad \text { as } m \rightarrow \infty .
$$

But this relation is readily seen to contradict (2.29).

CoROllary 3. Let $(g, h)$ vary in a family $M$ such that $\inf _{M} \gamma_{1}>0, \sup _{M} \gamma_{2}<0$, $\sup _{M}|g| \leqq$ const $<\infty$, $\sup _{M}|h| \leqq$ const $<\infty$, and $\inf _{M}(-1)^{i-1} h_{i}(x) \geqq \gamma_{3}>0$ for $x$ in some $\gamma_{4}$-neighborhood $V_{i}$ of $\partial_{i} G(i=1,2)$. Then there exists a $\delta_{0}$-neighborhood $W_{\delta_{0}}^{i}$ of $\partial_{i} G(i=1,2)$ such that $(-1)^{i-1} u(x, t) \geqq \varepsilon_{0}>0$ for $x \in W_{\delta_{0}}^{i} \cap \bar{G}, 0 \leqq t \leqq T$, and $\nabla_{x} u, \nabla_{x}^{2} u, D_{t} u$ are Hölder continuous on these sets; $\delta_{0}$ and $\varepsilon_{0}$ are independent of $(g, h)$ in $M$. 
Proof. From the proof of (2.8) it follows that $\varepsilon_{0}, \delta_{0}$ can be taken to be independent of $(g, h)$ in $M$. Now recall that $\left\{v_{m}\right\}$ is convergent to $u$ almost everywhere and also use Schauder-type estimates [1] for the $v_{m}$ in $\left[W_{\delta_{0}}^{i} \cap \bar{G}\right] \times[0, T]$.

THEOREM 4. Assume that (i)-(v) hold. Then there exists a unique weak solution of (1.1)-(1.5).

Proof. In case $c^{1} \equiv c^{2} \equiv 0$, an elegant proof of uniqueness was given in [4]. We shall extend it to the present case. If $u, v$ are two weak solutions, then

$$
\iint_{\Omega_{T}}[a(u)-a(v)]\left[\frac{\partial \phi}{\partial t}+e \Delta \phi+e \tilde{c} \phi\right] d x d t=0
$$

for every $\phi$ as in (1.9) where

and

$$
\begin{array}{rlrl}
e(x, t) & =\frac{u(x, t)-v(x, t)}{a(u(x, t))-a(v(x, t))} & & \text { if } u(x, t) \neq v(x, t), \\
& =0 & \text { if } u(x, t)=v(x, t),
\end{array}
$$

$$
\begin{aligned}
\tilde{c}(x, t) & =\frac{c(x, u) u-c(x, v) v}{u-v} & & \text { if } u \neq v, \\
& =0 & & \text { if } u=v .
\end{aligned}
$$

Noting that for $u \neq v$.

$$
\tilde{c}(x, t)=c(x, u)+\frac{c(x, u)-c(x, v)}{u-v} v
$$

and recalling the definition of $c(x, u)$, we easily conclude that $\tilde{c}(x, t)$ is a bounded function.

We can now proceed analogously to [4]. We approximate $e$ by a sequence of smooth functions $e_{m}$ (in [4] the notation of $c$ and $c_{m}$ are used instead of $e$ and $e_{m}$ ). We also approximate $\tilde{c}$ in $L^{2}\left(\Omega_{T}\right)$ by smooth $\tilde{c}_{m}$. For any $f$ in $C^{\infty}\left(\Omega_{T}\right)$ with compact support, we solve

$$
\begin{gathered}
\partial \phi_{m} / \partial t+e_{m} \Delta \phi_{m}+e{\tilde{c_{m}}}_{\phi_{m}}=f \text { in } \Omega_{T}, \\
\phi_{m}=0 \text { on } G(T) \text { and on } \partial G \times(0, T) .
\end{gathered}
$$

By multiplying the differential equation by $\Delta \phi_{m}$ and integrating, we find that

$$
\int_{G(\sigma)}\left|\nabla \phi_{m}\right|^{2} d x+\iint_{\Omega_{T}} e_{m}\left(\Delta \phi_{m}\right)^{2} d x d t \leqq A
$$

where $A$ is independent of $m$. If we now substitute $\phi=\phi_{m}$ into (2.30), then we find, by slightly modifying arguments given in [4], that

$$
I \equiv \iint_{\Omega_{T}}[a(u)-a(v)] f d x d t
$$


satisfies $|I| \leqq \varepsilon_{m}$ where $\varepsilon_{m} \rightarrow 0$ as $m \rightarrow \infty$. Hence $I=0$, from which it follows that $u=v$ almost everywhere.

To prove existence, let $\Psi$ be a continuous extension into $\bar{\Omega}_{T}$ of the function

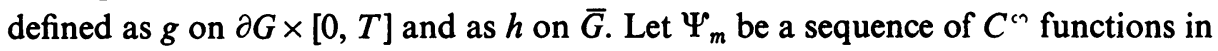
$\bar{\Omega}_{T}$ which converge uniformly to $\Psi$. For each $m$ there exists (by Theorem 3) a weak solution $u_{m}$ of (1.1)-(1.5), with the initial and boundary values given by $\Psi_{m}$. Since $\left\{u_{m}\right\},\left\{c\left(x, u_{m}\right)\right\}$ and $\left\{c\left(x, u_{m}\right) u_{m}\right\}$ are uniformly bounded sequences of functions in $\Omega_{T}$, we may assume (compare the proof of Theorem 3 ) that they all are convergent weakly in $L^{2}\left(\Omega_{T}\right)$ as well as almost everywhere. Denote by $u$ the weak limit of $\left\{u_{m}\right\}$. It follows that for every $\phi \in L^{2}\left(\Omega_{T}\right)$

$$
\lim _{m \rightarrow \infty} \iint_{\Omega_{T}} \phi(x, t) c\left(x, u_{m}\right) u_{m}(x, t) d x d t=\iint_{\Omega_{T}} \phi(x, t) c(x, u) u(x, t) d x d t .
$$

If we now write down (1.9) for $u=u_{m}$ and with $g, h$ replaced by $\Psi_{m}, \Psi_{m}$, and let $m \rightarrow \infty$, then we obtain the relation (1.9). Thus $u$ is a weak solution of (1.1)-(1.5).

RemarK 1. Note that from the uniqueness proof it follows that not only $u(x, t)$ but also $a(u(x, t))$ is uniquely determined almost everywhere.

REMARK 2 . The uniqueness proof clearly remains true also if the $c^{i}$ are functions of $(x, t)$. The use of Theorem 3 (in the existence proof) can be avoided if we replace $u_{m}$ by the solution of (2.5)-(2.7) with $g, h$ replaced by $\Psi_{m}$.

Suppose now that the assumptions of Theorem 4 hold for all $T>0$. Denoting by $u_{T}$ the solution in $\Omega_{T}$ and recalling that $u_{T}$ is a weak solution in $\Omega_{T^{\prime}}$ if $T^{\prime}<T$, it follows, by uniqueness, that $u_{T}=u_{T^{\prime}}$ in $\Omega_{T^{\prime}}$. Thus there exists a unique weak solution of (1.1)-(1.5) in $\Omega_{\infty}$.

\section{COROLlaRY 1. Corollary 3 to Theorem 3 holds for $T=\infty$.}

Indeed, this follows from the proof of that corollary.

We shall now draw some further conclusions in the case $T=\infty$. For simplicity we consider first the case $c^{1} \equiv c^{2} \equiv 0$.

Denote by $D_{x}$ the $(n-1)$-dimensional vector of tangential derivatives on $\partial G(t)$ (in terms of a fixed parametrization on $\partial G$ ). By $H_{\eta}(f ; t)$ we denote the Hölder coefficient of $f$ (with respect to the exponent $\eta$ ) on $\partial G(t)$.

We now give a better estimate on the constant $A^{\prime \prime}$ appearing in (2.12) than the one which results from the proof of Lemma 1 . Let us assume that

$$
\begin{aligned}
|g|+\left|D_{x} g\right|+\left|D_{t} g\right| \leqq A_{0} & \text { on } \partial G \times(0, \infty), \\
H_{n}\left(D_{x} g ; t\right) \leqq A_{0} & \text { for } 0<t<\infty,
\end{aligned}
$$

and consider the function $w=\partial v_{m} / \partial t$ on $\Omega_{i}^{* *}=\left(W_{\delta_{0}}^{i} \cap G\right) \times(1, \infty)$. It satisfies

$$
\alpha_{i} \partial w / \partial t=\Delta w \quad \text { in } \Omega_{i}^{* *}, \quad w=g_{t} \text { on } \partial_{i} G \times(1, \infty) .
$$

Denote by $\partial \Omega_{i}^{* *}$ the part of the boundary of $\Omega_{i}^{* *}$ which lies in $G \times(1, \infty)$, and denote by $\partial G_{i}^{*}$ the projection of $\partial \Omega_{i}^{* *}$ on $G(1)$. By decreasing $\delta_{0}$ if necessary, we may assume that $a_{m}^{\prime}\left(v_{m}\right)=\alpha_{i}$ in $V^{*} \times\left(\frac{1}{2}, \infty\right)$ where $V^{*}$ is a neighborhood of $\partial G_{i}^{*}$. 
But then one can conclude that $w=\partial v_{m} / \partial t$ is a bounded function on $\partial G_{i}^{*} \times(1, \infty)$. Using this result and (2.35), it follows, via the maximum principle, that $w$ is a bounded function in $\Omega_{i}^{* *}$.

We now consider, for fixed $t$, the system

$$
\begin{array}{rlrl}
\Delta v_{m} & =f & \text { in }\left(W_{\delta_{0}}^{i} \cap G\right) \times\{t\} & \left(f=\alpha_{i} \partial v_{m} / \partial t\right), \\
v_{m}=g & \text { on } \partial_{i} G(t) . &
\end{array}
$$

We can write $v_{m}=v_{m}^{\prime}+v_{m}^{\prime \prime}$ where $\Delta v_{m}^{\prime}=0, \Delta v_{m}^{\prime \prime}=f$ in $\left(W_{\delta_{0}}^{i} \cap G\right) \times\{t\}, v_{m}^{\prime}=g$ on $\partial_{i} G(t), v_{m}^{\prime}=v_{m}$ on $\partial G_{i}^{*} \times\{t\}$, and $v_{m}^{\prime \prime}=0$ on $\partial_{i} G(t)$ and on $\partial G_{i}^{*} \times\{t\}$. By results of [5], (2.12) on $\partial_{i} G \times(1, \infty)$ holds for $v_{m}^{\prime}$, with $A^{\prime \prime}$ depending on $A_{0}$ (in (2.34)) but not on $t$. Representing $v_{m}^{\prime \prime}$ in terms of Green's function and using standard estimates, we find that (2.12) holds also for $v_{m}^{\prime \prime}$, with $A^{\prime \prime}$ depending only on sup $|f|$. Since $f$ has already been estimated above, we conclude that the constant $A^{\prime \prime}$ in (2.12) is independent of $T$, i.e. (2.12) holds on $\partial G \times(0, \infty)$.

Using (2.13) we then easily obtain

Corollary 2. Assume that $c^{1} \equiv c^{2} \equiv 0$, that (i), (iii), (iv), (vi), (vii) hold for $T=\infty$, that (2.34) holds, and that

$$
\int_{0}^{\infty} \int_{\partial G}\left|g_{t}\right| d S_{x} d t<\infty
$$

Then

$$
\int_{0}^{t} \int_{G}\left|u_{t}\right|^{2} d x d t+\underset{0<t<\infty}{\operatorname{ess} \sup } \int_{G}|\nabla u(x, t)|^{2} d x<\infty .
$$

If $c^{i} \not \equiv$, then the assertion of Corollary 2 remains true (with slight modification in the proof).

REMARK 1. If $g_{1} \geqq 0, g_{2} \leqq 0$, then there occurs a difficulty in the proof of Theorem 3 , since the regions $\Omega_{i}^{*}$ cannot be constructed. One can, however, consider a modified problem with $g_{i}$ replaced by $g_{i \varepsilon}$ such that $(-1)^{i-1} g_{i \varepsilon}>0$ and $g_{i \varepsilon} \rightarrow g_{i}$ uniformly. From the family of solutions $u$ of the modified problems we can then choose a sequence which converges weakly and almost everywhere to a weak solution $u$.

If $h_{1} \geqq 0$ or $h_{2} \leqq 0$, then there occurs an additional difficulty in constructing $h_{i \varepsilon}$ satisfying the assumptions of Theorem 3 such that $a\left(h_{i \varepsilon}\right) \rightarrow a\left(h_{i}\right)$. There is no difficulty, however, in extending Theorem 4 (see $\S 6$ ).

3. Some properties of solutions. We prove a comparison theorem.

THEOREM 5. Assume that the conditions of Theorem 4 hold for $g, h$ and for another pair $\hat{g}, \hat{h}$. Denote by $u$ and $\hat{u}$ the corresponding weak solutions. If $\hat{g} \geqq g, \hat{h} \geqq h$, then $\hat{u} \geqq u$ almost everywhere.

Proof. Since $\hat{g}-g$ and $\hat{h}-h$ form a continuous nonnegative function on $G \cup[\partial G \times[0, T]]$, we can extend them to a continuous function $N(x, t)$ in $\bar{\Omega}_{T}$ which is also nonnegative (see [3], [7]). Let $\left\{\Psi_{j}\right\}$ and $\left\{N_{j}\right\}$ be sequences of smooth 
functions in $\bar{\Omega}_{T}$ which approximate $\Psi$ and $N$ uniformly in $\bar{\Omega}_{T}$. We can take the $N_{j}$ to be positive. Set $\hat{\Psi}_{j}=\Psi_{j}+N_{j}$. Denote by $u_{j}, \hat{u}_{j}$ the weak solutions of (1.1)-(1.5) corresponding to the data $\Psi_{j}, \hat{\Psi}_{j}$ respectively.

Each $u_{j}$ is the pointwise limit (almost everywhere) of a sequence $\left\{v_{j m}\right\}$, where $v_{j m}$ satisfies (2.5)-(2.7) with $g, h$ replaced by $\Psi_{j}$. Next, $\hat{u}_{j}$ is the pointwise limit of a sequence of solutions $\hat{v}_{j m}$ of (2.5)-(2.7) with $g, h$ replaced by $\hat{\Psi}_{j}$ and $L^{m} v$ replaced by $L^{m} v+1 / m$. Since $\hat{\Psi}_{j}>\Psi_{j}$, the Lemma of Westphal-Prodi (see [1, p. 52]) shows that $\hat{v}_{j m}>v_{j m}$ in $\Omega_{T}$. Hence $\hat{u}_{j} \geqq u_{j}$ almost everywhere.

By the proof of Theorem 4, $u$ and $\hat{u}$ are the pointwise limits (almost everywhere) of subsequences (with the same indices) of $\left\{u_{j}\right\}$ and $\left\{\hat{u}_{j}\right\}$ respectively. The assertion now follows.

Theorem 5 , under stronger smoothness restrictions on $g, h, \hat{g}, \hat{h}$, was proved (by a different method) in [4].

THEOREM 6. Let the assumptions of Theorem 3 hold. In any open subset $M$ of $\Omega_{T}$ where $u \geqq 0$ and $a(u) \geqq 0(u \leqq 0$ and $a(u) \leqq-\alpha), u$ is a classical solution of $\alpha_{1} \partial u / \partial t=L_{1} u\left(\alpha_{2} \partial u / \partial t=L_{2} u\right)$.

Proof. In $M, u$ is a weak solution of $\alpha_{1} u_{t}-L_{1} u=0$. Since $u \in W^{1,2}\left(\Omega_{T}\right)$, it is also a "strong" solution. From [6] it follows that $u$ is Hölder continuous, and it is then easy to see that $u$ is a classical solution of $\alpha_{1} u_{t}-L_{1} u=0$.

REMARK. If $c(x, t) \equiv 0$, then the assertion of Theorem 6 is obviously valid even under the assumptions of Theorem 4.

4. Stability of solutions. In this and in the following section, we assume, for simplicity, that $c^{1} \equiv c^{2} \equiv 0$. Denote by $H_{\eta}(k)$ the Hölder coefficient of a function $k$ (in a set to be specified) with respect to the exponent $\eta$.

Let $M$ be a family of functions ( $g, h$ ) satisfying (iii), (iv), (vi), (vii), and let

$$
g_{1} \geqq \bar{\gamma}_{1}>0, \quad g_{2} \leqq \bar{\gamma}_{2}<0, \quad(-1)^{i-1} h_{i}(x) \geqq \bar{\gamma}_{3}
$$

in a $\delta$-neighborhood $V_{i}$ of $\partial_{i} G(i=1,2)$,

$$
|h| \leqq \bar{A}, \quad|g| \leqq \bar{A},
$$

for all $(g, h)$ in $M$, where $\bar{\gamma}_{1}, \bar{\gamma}_{2}, \bar{\gamma}_{3}, \delta, \bar{A}$ are independent of $(g, h)$.

Take any two elements $(g, h)$ and $(\hat{g}, \hat{h})$ in $M$ and denote by $u, \hat{u}$ the corresponding weak solutions of (1.1)-(1.5). Denote by $\hat{\Psi}$ the function $\Psi$ which occurs in (vii) when $g=\hat{g}, h=\hat{h}$.

THEOREM 7. Under the foregoing assumptions, there exists a constant $B$ depending only on $\bar{\gamma}_{1}, \bar{\gamma}_{2}, \bar{\gamma}_{3}, \bar{\delta}, \bar{A}, \partial G, \alpha_{1}, \alpha_{2}, \alpha$ such that

$$
\begin{aligned}
& \iint_{\Omega_{T}}(\hat{u}-u)[a(\hat{u})-a(u)] d x d t \\
& \quad \leqq B \int_{G}|\hat{h}-h|^{2} d x+B \iint_{\Omega_{T}}|\hat{\Psi}-\Psi|^{2} d x d t+B \iint_{\Omega_{T}}\left|\nabla_{x} \hat{\Psi}-\nabla_{x} \Psi\right|^{2} d x d t .
\end{aligned}
$$


Proof. We shall denote various positive constants depending only on $\bar{\gamma}_{1}, \bar{\gamma}_{2}, \bar{\gamma}_{3}$, $\bar{\delta}, \bar{A}, \partial G, \alpha_{1}, \alpha_{2}, \alpha$ by $B$. Set

$$
\begin{aligned}
& e(x, t)=(\hat{u}-u) /(a(\hat{u})-a(u)) \text { if } \hat{u} \neq u \text {, } \\
& =0 \quad \text { if } \hat{u}=u \text {. }
\end{aligned}
$$

Then $0 \leqq e \leqq 1 / \alpha_{3}$. Choose a sequence $\left\{\bar{e}_{m}\right\}$ of nonnegative $C^{\infty}$ functions in $\bar{\Omega}_{T}$ such that $\left\|\bar{e}_{m}-e\right\| \leqq 1 / m$ (" $\|\cdot\|$ " means the $L^{2}\left(\Omega_{T}\right)$-norm). If we take the $\bar{e}_{m}$ to be appropriate mollifiers of $c$ (see [1]) then we have $\bar{e}_{m} \leqq 1+1 / \alpha_{3}$. It easily follows that the functions $e_{m}=\bar{e}_{m}+1 / m$ satisfy

$$
\begin{aligned}
e_{m} & \leqq 2+1 / \alpha_{3}, \\
\left\|e_{m}-e\right\| & \rightarrow 0, \\
\left\|e / e_{m}\right\| & \leqq B\left(1+T^{1 / 2}\right) .
\end{aligned}
$$

Let $f$ be any function in $C^{\infty}\left(\bar{\Omega}_{T}\right)$ and consider the system:

$$
\begin{array}{rlrl}
\partial \phi_{m} / \partial t+e_{m} \Delta \phi_{m} & =f & & \text { in } \Omega_{T}, \\
\phi_{m}=0 & & \text { on } \partial G \times(0, T), \\
\phi_{m}=0 & & \text { on } G(T) .
\end{array}
$$

Since (1.9) holds with $\phi=\phi_{m}$ for both $u, g, h$ and $\hat{u}, \hat{g}, \hat{h}$, we get, by subtraction,

$$
\begin{array}{r}
\iint_{\Omega_{T}}[a(\hat{u})-a(u)]\left[\frac{\partial \phi_{m}}{\partial t}+e_{m} \Delta \phi_{m}\right] d x d t=\iint_{\Omega_{T}}[a(\hat{u})-a(u)]\left(e_{m}-e\right) \Delta \phi_{m} d x d t \\
\quad+\int_{0}^{T} \int_{\partial G}(\hat{g}-g) \frac{\partial \phi_{m}}{\partial \nu} d S_{x} d t-\int_{G(0)}[a(\hat{h})-a(h)] \phi_{m} d x \equiv I_{m}+J_{m}+K_{m} .
\end{array}
$$

Multiply both sides of (4.8) by $\Delta \phi_{m}$ and integrate over $\Omega_{T}-\Omega_{\sigma}$. Integrating by parts and using (4.9), (4.10), and the inequality $2 \iint\left|f \Delta \phi_{m}\right| \leqq \iint\left(f^{2} / e_{m}\right)+\iint e_{m}\left|\Delta \phi_{m}\right|$, we find that

$$
\int_{G(\sigma)}\left|\nabla \phi_{m}\right|^{2} d x+\frac{1}{2} \int_{\sigma}^{T} \int_{G} e_{m}\left|\Delta \phi_{m}\right|^{2} d x d t \leqq \frac{1}{2} \int_{\sigma}^{T} \int_{G} \frac{f^{2}}{e_{m}} d x d t
$$

Since $\phi_{m}=0$ on $\partial G(\sigma)$, we also have

$$
\int_{G(\sigma)} \phi_{m}^{2} d x \leqq B \int_{G(\sigma)}\left|\nabla \phi_{m}\right|^{2} d x \leqq B \int_{\sigma}^{T} \int_{G} \frac{f^{2}}{e_{m}} d x d t
$$

Using Schwarz's inequality and (4.13), it follows that

$$
\left(K_{m}\right)^{2} \leqq B\left(\int_{G}|\hat{h}-h|^{2} d x\right) \iint_{\Omega_{T}} \frac{f^{2}}{e_{m}} d x d t .
$$

Let $p(x)$ be a nonnegative $C^{\infty}$ function in $\bar{G}$ which is equal to 1 on $\partial G$ and which 
vanishes outside $W_{\delta}^{i} \cap G(i=1,2) . W_{\delta}^{i}$ is a $\delta$-neighborhood of $\partial_{i} G$, and we take $\delta=\delta_{0} / 2$ where $\delta_{0}$ is the constant appearing in Corollary 3 to Theorem 3 .

Next, we write

$$
\begin{aligned}
\left|J_{m}\right| & =\left|\int_{0}^{T} \int_{\partial G}(p \hat{\Psi}-p \Psi) \frac{\partial \phi_{m}}{\partial \nu} d S_{x} d t\right| \\
& \leqq\left|\iint_{\Omega_{T}} p(\hat{\Psi}-\Psi) \Delta \phi_{m} d x d t\right|+\left|\iint_{\Omega_{T}} \nabla(p \hat{\Psi}-p \Psi) \cdot \nabla \phi_{m} d x d t\right| \equiv J_{m}^{1}+J_{m}^{2} .
\end{aligned}
$$

In both $J_{m}^{1}$ and $J_{m}^{2}$ the integrand vanishes outside $\Omega^{1}$ and $\Omega^{2}$ respectively, where $\Omega^{i}=\left(W_{\delta}^{i} \cap G\right) \times(0, T)$. If, on the other hand, $(x, t) \in \Omega^{i}$, then $(-1)^{i-1} \hat{u}>0$, $(-1)^{i-1} u>0$, and these inequalities persist, in fact, also in a $\left(\delta_{0} / 2\right)$-neighborhood of $\Omega^{i}$. Thus, in that neighborhood we have $e=\alpha_{i}$. Hence (recalling that $\bar{e}_{m}$ is a mollifier of $e$ ), $e_{m} \geqq \alpha_{i}$ in $\Omega^{i}$ provided $m \geqq m_{0}$.

We now have

$$
\begin{aligned}
\left(J_{m}^{1}\right)^{2} & \leqq B\left[\iint_{\Omega_{T}} p(\hat{\Psi}-\Psi)^{2} d x d t\right] \iint_{\Omega^{1} \cup \Omega^{2}} p e_{m}\left(\Delta \phi_{m}\right)^{2} d x d t \\
& \leqq B\left[\iint_{\Omega_{T}}(\hat{\Psi}-\Psi)^{2} d x d t\right] \iint_{\Omega_{T}} e_{m}\left(\Delta \phi_{m}\right)^{2} d x d t
\end{aligned}
$$

Next,

$$
\left(J_{m}^{2}\right)^{2} \leqq B\left\{\iint_{\Omega_{T}}\left[(\hat{\Psi}-\Psi)^{2}+|\nabla \hat{\Psi}-\nabla \Psi|^{2}\right] d x d t\right\} \iint_{\Omega_{T}}\left|\nabla \phi_{m}\right|^{2} d x d t
$$

Using (4.12) to evaluate the last integrals in (4.15), (4.16), and combining the resulting inequalities, we conclude that

$$
\left(J_{m}\right)^{2} \leqq B\left\{\iint_{\Omega_{T}}\left[(\hat{\Psi}-\Psi)^{2}+|\nabla \hat{\Psi}-\nabla \Psi|^{2}\right] d x d t\right\} \iint_{\Omega_{T}} \frac{f^{2}}{e_{m}} d x d t
$$

It remains to estimate $I_{m}$.

$$
\begin{aligned}
\left|I_{m}\right| & \leqq B \iint_{\Omega_{T}}\left|e_{m}-e\right|\left|\Delta \phi_{m}\right| d x d t=B \iint_{\Omega_{T}}\left(\left(e_{m}\right)^{1 / 2}+e^{1 / 2}\right)\left|\left(e_{m}\right)^{1 / 2}-e^{1 / 2}\right|\left|\Delta \phi_{m}\right| d x d t \\
& =B \iint_{\Omega_{T}}\left(e_{m}\right)^{1 / 2}\left|\left(e_{m}\right)^{1 / 2}-e^{1 / 2}\right|\left|\Delta \phi_{m}\right| d x d t+B \iint_{\Omega_{T}} e^{1 / 2}\left|\left(e_{m}\right)^{1 / 2}-e^{1 / 2}\right|\left|\Delta \phi_{m}\right| d x d t \\
& \equiv I_{m}^{1}+I_{m}^{2} .
\end{aligned}
$$

Given $\eta>0$, let $E_{\eta}=\left\{(x, t) \in \Omega_{T} ;\left|\left(e_{m}\right)^{1 / 2}-e^{1 / 2}\right|>\eta\right\}$. Since $e_{m} \rightarrow e$ in measure, for any $\lambda>0$ there exists an $m_{1}=m_{2}(\eta, \lambda)$ such that meas. $\left(E_{\eta}\right)<\lambda$ if $m \geqq m_{1}$. Setting $F_{\eta}=\Omega_{T}-E_{\eta}$ and using (4.5), we have

$$
I_{m}^{1} \leqq B \iint_{F_{\eta}}+B \iint_{E_{\eta}} \leqq B \eta \iint_{F_{\eta}}\left(e_{m}\right)^{1 / 2}\left|\Delta \phi_{m}\right| d x d t+B \iint_{E_{\eta}}\left(e_{m}\right)^{1 / 2}\left|\Delta \phi_{m}\right| d x d t
$$


Hence, by Schwarz's inequality and (4.12),

$$
\begin{aligned}
\left(I_{m}^{1}\right)^{2} & \leqq B \eta^{2} \iint_{\Omega_{T}} e_{m}\left(\Delta \phi_{m}\right)^{2} d x d t+B \lambda \iint_{E_{\eta}} e_{m}\left(\Delta \phi_{m}\right)^{2} d x d t \\
& \leqq B\left(\eta^{2}+\lambda\right) \iint_{\Omega_{T}} \frac{f^{2}}{e_{m}} d x d t .
\end{aligned}
$$

Next,

$$
I_{m}^{2} \leqq B \eta \iint_{F_{\eta}} e^{1 / 2}\left|\Delta \phi_{m}\right| d x d t+B \iint_{E_{\eta}} e^{1 / 2}\left|\Delta \phi_{m}\right| d x d t .
$$

Writing $e^{1 / 2}=\left(e_{m}\right)^{1 / 2}\left(e^{1 / 2} /\left(e_{m}\right)^{1 / 2}\right)$ and using Schwarz's inequality and (4.12), we get

$$
\left(I_{m}^{2}\right)^{2} \leqq\left(B \eta^{2} \iint_{\Omega_{T}} \frac{e}{e_{m}} d x d t+B \iint_{\Sigma_{\eta}} \frac{e}{e_{m}} d x d t\right) \iint_{\Omega_{T}} \frac{f^{2}}{e_{m}} d x d t
$$

Since, by Schwarz's inequality and (4.7),

$$
\iint_{\Omega_{T}} \frac{e}{e_{m}} d x d t \leqq B T^{1 / 2}\left\|\frac{e}{e_{m}}\right\| \leqq B T, \quad \iint_{E_{\eta}} \frac{e}{e_{m}} d x d t \leqq B(\lambda T)^{1 / 2}
$$

we conclude that

$$
\left(I_{m}^{2}\right)^{2} \leqq\left(B T \eta^{2}+B(T \lambda)^{1 / 2}\right) \iint_{\Omega_{T}} \frac{f^{2}}{e_{m}} d x d t .
$$

Combining (4.19) with (4.18), we have

$$
\left(I_{m}\right)^{2} \leqq\left[B(1+T) \eta^{2}+B(T \lambda)^{1 / 2}+B \lambda\right] \iint_{\Omega_{T}} \frac{f^{2}}{e_{m}} d x d t .
$$

We now combine the estimates (4.20), (4.17), (4.14) and find, from (4.11), (4.8),

$$
\left|\iint_{\Omega_{T}}[a(\hat{u})-a(u)] f d x d t\right|^{2} \leqq B\left[(1+T) \eta^{2}+(T \lambda)^{1 / 2}+\lambda+N\right] \iint_{\Omega_{T}} \frac{f^{2}}{e_{m}} d x d t
$$

where, for brevity, we have denoted by $N$ the sum of the three integrals on the right-hand side of (4.3).

We have proved (4.21) for any $f \in C^{\infty}\left(\bar{\Omega}_{T}\right)$. Now let $f$ be any function in $L^{2}\left(\Omega_{T}\right)$ and construct a sequence of functions $f_{j}$ in $C^{\infty}\left(\bar{\Omega}_{T}\right)$ such that $\left\|f-f_{j}\right\| \rightarrow 0$. Then (4.21) holds for $f=f_{j}$ (with $B$ independent of $f_{j}$ ). Hence the inequality holds also for $f$.

Taking, in particular, $f=\hat{u}-u$, and denoting the left-hand side of (4.3) by $\rho$, we get

$$
\rho^{2} \leqq B\left[(1+T) \eta^{2}+(T \lambda)^{1 / 2}+\lambda+N\right] \iint_{\Omega_{T}} \frac{f^{2}}{e_{m}} d x d t \quad(f=\hat{u}-u)
$$

provided $m \geqq m_{2}\left(m_{2}\right.$ may depend on $\left.u, \hat{u}, T\right)$. 
LEMMA 2. If $f=\hat{u}-u$, then

$$
\lim _{m \rightarrow \infty} \iint_{\Omega_{T}} \frac{f^{2}}{e_{m}} d x d t=\iint_{\Omega_{T}} \frac{f^{2}}{e} d x d t
$$

where, by definition, $f^{2} / e=0$ whenever $\hat{u}=u$ (i.e. wherever $e=0$ ).

Suppose the lemma is true. Then, letting $m \rightarrow \infty$ in (4.22), we obtain

$$
\begin{aligned}
\rho^{2} & \leqq B\left[(1+T) \eta^{2}+(T \lambda)^{1 / 2}+\lambda+N\right] \iint_{\Omega_{T}} \frac{(\hat{u}-u)^{2}}{e} d x d t \\
& =B\left[(1+T) \eta^{2}+(T \lambda)^{1 / 2}+\lambda+N\right] \rho .
\end{aligned}
$$

Since $\eta$ and $\lambda$ are arbitrary positive numbers, it follows that $\rho \leqq B N$, which is precisely the inequality (4.3).

Proof of Lemma 2. In the following proof, the constants $B$ may depend also on $T$. For any $\delta>0$, set

$$
Q_{\delta}=\left\{(x, t) \in \Omega_{T} ;|\hat{u}(x, t)-u(x, t)|<\delta\right\}, \quad P_{\delta}=\Omega_{T}-Q_{\delta} .
$$

Write

$$
\iint_{\Omega_{T}} \frac{f^{2}}{e_{m}} d x d t=\iint_{Q_{\delta}} \frac{f^{2}}{e_{m}} d x d t+\iint_{P_{\delta}} \frac{f^{2}}{e_{m}} d x d t
$$

and set $\bar{Q}_{\delta}=Q_{\delta}-\left\{(x, t) \in \Omega_{T} ; \hat{u}(x, t)=u(x, t)\right\}$. We have

$$
\begin{aligned}
& \iint_{Q_{0}} \frac{f^{2}}{e_{m}} d x d t=\iint_{\bar{Q}_{\delta}} \frac{(\hat{u}-u)^{2}}{e_{m}} d x d t \\
& =\iint_{\bar{Q}_{0}} \frac{\hat{u}-u}{a(\hat{u})-a(u)} \frac{a(\hat{u})-a(u)}{e_{m}}(\hat{u}-u) d x d t \\
& \leqq B \iint_{\bar{Q}_{\delta}} \frac{e}{e_{m}}|\hat{u}-u| d x d t \leqq B \delta \iint_{\bar{Q}_{\delta}} \frac{e}{e_{m}} d x d t \leqq B \delta,
\end{aligned}
$$

where (4.7) has been used. We also have

$$
\begin{aligned}
\iint_{Q_{\delta}} f^{2} d x d t & =\iint_{\bar{Q}_{\delta}} \frac{(\hat{u}-u)^{2}}{e} d x d t=\iint_{\bar{Q}_{\delta}}[a(\hat{u})-a(u)](\hat{u}-u) d x d t \\
& \leqq B \iint_{Q_{\delta}}|\hat{u}-u| d x d t \leqq B \delta
\end{aligned}
$$

$P_{\delta}$ is a measurable set. Hence almost every point $(x, t)$ of $P_{\delta}$ is a density point, i.e.

$$
\lim _{\delta \rightarrow 0} \frac{\mu\left(P_{\delta} \cap K_{\varepsilon}\right)}{\mu\left(K_{\varepsilon}\right)}=1
$$

where $K_{\varepsilon}$ is a ball with center $(x, t)$ and radius $\varepsilon$ and $\mu$ is the Lebesgue measure. Set

$$
E_{m}=\left\{(x, t) ; \mu\left(P_{\delta} \cap K_{\varepsilon}\right) \geqq \frac{1}{2} \mu\left(K_{\varepsilon}\right) \text { for all } 0<\varepsilon<1 / m\right\}
$$


Then $E_{m} \subset E_{m+1}$ and $\mu\left(P_{\delta}-P_{\delta}^{0}\right)=0$ where $P_{\delta}^{0}=\bigcup_{m=1}^{\infty} E_{m}$. It follows that for any $\varepsilon>0$ there exists a $j=j(\varepsilon)$ such that $\mu\left(P_{\delta}-E_{j}\right)<\varepsilon$. Hence (compare (4.24)),

$$
\iint_{P_{\delta}-E_{j}} \frac{f^{2}}{e_{m}} d x d t \leqq B \iint_{P_{\delta}-E_{j}} \frac{e}{e_{m}} d x d t \leqq B \varepsilon^{1 / 2}
$$

where (4.7) has been used. Similarly,

$$
\iint_{P_{\delta}-E} \frac{f^{2}}{e} d x d t \leqq B \varepsilon^{1 / 2}
$$

Note next that $e(x, t) \geqq \lambda(\delta)$ on $P_{\delta}$ where $\lambda(\delta)>0, \lambda(\delta) \rightarrow 0$ if $\delta \rightarrow 0$. Since $e \geqq 0$ in $\Omega_{T}$ and $\bar{e}_{m}$ is a mollifier of $e$, it follows that $e_{m} \geqq \bar{e}_{m}>\lambda(\delta) / 2$ on $E_{j}$, provided $m$ is sufficiently large. Thus $0 \leqq f^{2} / e_{m} \leqq B$ in $E_{j}$. Since $e_{m} \rightarrow e$ almost everywhere, the Lebesgue Bounded Convergence Theorem yields

$$
\lim _{m \rightarrow \infty} \iint_{E_{j}} \frac{f^{2}}{e_{m}} d x d t=\iint_{E_{j}} \frac{f^{2}}{e} d x d t
$$

Combining (4.23)-(4.28), we find that

$$
\underset{m \rightarrow \infty}{\limsup }\left|\iint_{\Omega_{T}} \frac{f^{2}}{e_{m}} d x d t-\iint_{\Omega_{T}} \frac{f^{2}}{e} d x d t\right| \leqq B\left(\delta+\varepsilon^{1 / 2}\right) .
$$

Since $\delta$ and $\varepsilon$ are arbitrary positive numbers, the proof of the lemma is complete.

COROLLARY 1. Under the assumption of Theorem 7, if $T>2$,

$$
\begin{aligned}
& \iint_{\Omega_{T}}(\hat{u}-u)[a(\hat{u})-a(u)] d x d t \\
& \leqq B \int_{G}(\hat{h}-h)^{2} d x+B \int_{0}^{2} \int_{G}\left[(\Psi-\Psi)^{2}+\left|\nabla_{x} \Psi-\nabla_{x} \Psi\right|^{2}\right] d x d t \\
& \quad+B \int_{1}^{T} \int_{\partial G}\left[(\hat{g}-g)^{2}+\left|D_{x} \hat{g}-D_{x} g\right|^{2}\right] d S_{x} d t
\end{aligned}
$$

Proof. We first construct an extension $\Psi^{*}$ of $\hat{g}-g$ into $\Omega_{T}-\Omega_{1}$, such that

$$
\int_{1}^{T} \int_{G}\left[\left(\Psi^{*}\right)^{2}+\left|\nabla_{x} \Psi^{* *}\right|^{2}\right] d x d t \leqq B \int_{1}^{T} \int_{\partial G}\left[(\hat{g}-g)^{2}+\left|D_{x} \hat{g}-D_{x} g\right|^{2}\right] d S_{x} d t
$$

To do this, we introduce a family of parallel surfaces $\partial G_{\varepsilon}\left(\partial G_{0}=\partial G, \partial G_{\varepsilon} \subset \partial G_{\varepsilon^{\prime}}\right.$ if $\left.\varepsilon>\varepsilon^{\prime}\right)$ for $0 \leqq \varepsilon \leqq \varepsilon_{0}$ and orthogonal curves $l_{\mu}(\sigma)$, where $\mu$ is the $(n-1)$-dimensional parameter on $\partial G$ and $l_{\mu}(\varepsilon) \in \partial G_{\varepsilon}$. Next we introduce a $C^{\infty}$ function $\zeta(\sigma)$ with $\zeta(0)=1, \zeta(\varepsilon)=0$ for $\varepsilon \geqq \varepsilon_{0}$. If we now define $\Psi^{*}$ at $l_{\mu}(\varepsilon)$ to be equal $\zeta(\varepsilon)$ times $\hat{g}-g$ at the point $l_{\mu}(0)$, then we obtain the desired extension.

Let $\lambda_{1}, \lambda_{2}$ be $C^{\infty}$ functions in $\bar{\Omega}_{T}$ such that $\lambda_{1}=0$ if $t>2, \lambda_{2}=0$ if $t<1$, and $\lambda_{1}+\lambda_{2} \equiv 1$ in $\Omega_{T}$. If we use $\lambda_{1}(\Psi-\Psi)+\lambda_{2} \Psi^{*}$ instead of $\Psi-\Psi$ in the proof of Theorem 7, then we obtain (4.29). 
Since $(\hat{u}-u)[a(\hat{u})-a(u)] \geqq \alpha_{3}(\hat{u}-u)^{2}$ where $\alpha_{3}=\min \left(\alpha_{1}, \alpha_{2}\right)$, we get

Corollary 2. Under the assumptions of Theorem 7, the integral $\iint_{\Omega_{T}}(\hat{u}-u)^{2} d x d t$ is bounded by the right-hand sides of both (4.3) and (4.29).

We give an illustration of Corollary 2. Let $u$ be a classical solution of (1.1)-(1.5) and let $\hat{u}$ be a weak solution with data $\hat{h}, \hat{g}$ satisfying

$$
\int_{G}|\hat{h}-h|^{2} d x+\int_{0}^{T} \int_{G}\left[(\Psi-\Psi)^{2}+\left|\nabla_{x} \Psi-\nabla_{x} \Psi\right|^{2}\right] d x d t \leqq \varepsilon^{2}
$$

Assume also that $(g, h),(\hat{g}, \hat{h})$ belong to a class $M$ as in Theorem 7 . Then

$$
\iint_{\Omega_{T}}|\hat{u}-u|^{2} d x d t \leqq B^{2} \varepsilon^{2}
$$

Now denote by $S_{0}$ some (small) neighborhood of the free boundary of $u$ in $\Omega_{T}$. Then $|u| \geqq \rho>0$ in $D_{0}=\Omega_{T}-S_{0}$. It follows that $\hat{u} \neq 0$ at all the points of $D_{0}$ with the exception of a set $S_{*}$ of measure $\leqq B \varepsilon / \rho$. By Theorem $6, \hat{u}$ is a classical solution in $\Omega_{T}-\left(S_{0} \cup S_{*}\right)$.

REMARK. If $c^{i} \not \equiv 0$, then one can still prove Theorem 7 and its corollaries, but the constants $B$ depend, in general, on $T$. Thus, instead of (4.8) one considers the equation

$$
\partial \phi_{m} / \partial t+e_{m} \Delta \phi_{m}+e_{m} \tilde{c}_{m} \phi_{m}=f \text { in } \Omega_{T}
$$

where $\left\{\tilde{c}_{m}\right\}$ is a sequence of smooth functions which converge in $L^{2}\left(\Omega_{T}\right)$ to the bounded function $\tilde{c}$ given by (2.32) (or (2.33)) when $v=\hat{u}$.

5. Asymptotic behavior of solutions. We shall need the following assumption:

(viii) $g_{\infty}(x)$ is a function in $C^{2+\eta}(\partial G)$, positive on $\partial_{1} G$ and negative on $\partial_{2} G$.

Denote by $w$ the solution of

$$
\Delta w=0 \quad \text { in } G, \quad w=g_{\infty} \text { on } \partial G .
$$

Then $w$ satisfies

$$
\iint_{\Omega_{T}}\left[a(w) \frac{\partial \phi}{\partial t}+w \Delta \phi\right] d x d t=\int_{0}^{T} \int_{\partial G} g_{\infty} \frac{\partial \phi}{\partial \nu} d S_{x} d t-\int_{G} a(w) \phi d x
$$

for any $\phi$ as in (1.9). Applying Corollary 1 to Theorem 7 with $\hat{u}=w$ and with $T \rightarrow \infty$, we obtain the following.

THEOREM 8. Let (i), (iii), (iv), (vi), (vii), (viii) hold for $T=\infty$ and assume that $g$ is a bounded function on $\partial G \times[0, \infty)$. Assume also that

$$
N \equiv \int_{1}^{\infty} \int_{\partial G}\left[\left(g-g_{\infty}\right)^{2}+\left|D_{x} g-D_{x} g_{\infty}\right|^{2}\right] d S_{x} d t<\infty
$$


Denoting by $w$ the solution of (5.1), we have

$$
\begin{aligned}
\int_{0}^{\infty} \int_{G}(u-w) & {[a(u)-a(w)] d x d t } \\
& \leqq B N+B \int_{G}(w-h)^{2} d x+B \int_{0}^{2} \int_{G}\left[(w-\Psi)^{2}+|\nabla w-\nabla \Psi|^{2}\right] d x d t
\end{aligned}
$$

where $B$ is a constant. If $(g, h)$ varies in a family $M$ as in Theorem 7 , then $B$ depends only on $\bar{\gamma}_{1}, \bar{\gamma}_{2}, \bar{\gamma}_{3}, \delta, \bar{A}, \partial G, \alpha_{1}, \alpha_{2}, \alpha$.

COROLlaRY. Under the assumptions of Theorem 8,

$$
\int_{0}^{\infty} \int_{G}|u(x, t)-w(x)|^{2} d x d t<\infty .
$$

THEOREM 9. Let the assumptions of Theorem 8 hold and, in addition, assume that (2.34), (2.36) hold. Then

$$
\int_{G}|u(x, t)-w(x)|^{2} d x \rightarrow 0 \quad \text { as } \quad t \rightarrow \infty .
$$

Proof. From Corollary 2 to Theorem 4 we have

$$
\int_{0}^{\infty} \int_{G}\left|u_{t}(x, t)\right|^{2} d x d t<\infty
$$

Set $v=u-w$. If (5.6) is false, then there exists a sequence $\left\{\sigma_{m}\right\} \rightarrow \infty$ such that

$$
\int_{G}\left|v\left(x, \sigma_{m}\right)\right|^{2} d x \geqq \mu>0 \text { for all } m \text {. }
$$

We may assume that $\sigma_{m+1}-\sigma_{m} \geqq 1$. Writing

$$
v(x, t)=v\left(x, \sigma_{m}\right)+\int_{\sigma_{m}}^{t} v_{\tau}(x, \tau) d \tau
$$

we get

$$
\int_{G}\left|v\left(x, \sigma_{m}\right)\right|^{2} d x \leqq 2 \int_{G}|v(x, t)|^{2} d x+2 \int_{G}\left|\int_{\sigma_{m}}^{t} v_{\tau}(x, \tau) d \tau\right|^{2} d x .
$$

If $0 \leqq t-\sigma_{m} \leqq 1$, then, by Schwarz's inequality, the last integral is bounded by

$$
\int_{\sigma_{m}}^{t} \int_{G}\left|v_{\tau}(x, \tau)\right|^{2} d x d \tau \leqq \varepsilon_{m}
$$

where, in view of (5.7), $\varepsilon_{m} \rightarrow 0$ as $m \rightarrow \infty$.

It follows from (5.8)-(5.10) that

$$
\int_{G}|v(x, t)|^{2} d x \geqq \frac{\mu}{4} \text { for } \sigma_{m} \leqq t \leqq \sigma_{m}+1
$$

provided $m \geqq m_{0}$. This, however, contradicts (5.5). 
As is well known (see, for instance, [11]), if $2<p<2 n /(n-2)$ when $n \geqq 2$ and $2<p \leqq \infty$ when $n=1$, then the inequality

$$
\|\phi\|_{L^{p}(G)}<\frac{K}{\delta^{\lambda}}\|\phi\|_{L^{2}(G)}+\delta^{1-\lambda}\|\nabla \phi\|_{L^{2}(G)}
$$

holds for any $\phi \in W^{1,2}(G)$ and all $0<\delta<\delta_{0}$, where $K, \delta_{0}, \lambda$ are positive constants and $\lambda<1$. Using this relation for $\phi(x)=u(x, t)-w(x)$ and employing (5.6) and (2.21) (which holds with $B$ independent of $T$ ), we easily get

COROLlARY. If $n \geqq 2$ and $p<2 n /(n-2)$, then

$$
\int_{G}|u(x, t)-w(x)|^{p} d x \rightarrow 0 \text { as } t \rightarrow \infty
$$

If $n=1$, then

$$
u(x, t) \rightarrow w(x) \text { as } t \rightarrow \infty,
$$

uniformly with respect to $x$ in $\bar{G}$.

6. Generalizations. We first extend Theorem 4 to very general data.

Definition. Let $h(x)$ be any bounded measurable function on $\bar{G}$ and let $\beta(x)$ be a measurable function defined as $a(h(x))$ if $h(x) \neq 0$ and satisfying $-\alpha \leqq \beta(x) \leqq 0$ at the points where $h(x)=0$. We then write $a(h)$ (or $a(h(x))$ ) for the function $\beta(x)$. We now define a weak solution of (1.1)-(1.5) with $a(u)=a(h)$ on $t=0$ by the condition (1.9).

For example, we note that Corollary 1 to Theorem 3 in fact asserts that for any $0<\sigma<T$, the weak solution of (1.1)-(1.5) is also a weak solution for $\sigma<t<T$ with $a(u)=a(k)$ on $t=\sigma$, where $k(x)=u(x, \sigma)$.

Note that $a(h)$ determines $h(x)$ uniquely.

THEOREM 10. Assume that (i), (ii) hold and let $g, h, a(h)$ be any bounded measurable functions. Then there exists $a$ unique weak solution of $(1.1)-(1.5)$ with $a(u)=a(h)$ on $t=0$.

Proof. Uniqueness follows from Theorem 4. To prove existence, define functions $\hat{h}_{m}$ by $a_{m}\left(\hat{h}_{m}\right)=a(h)$. The $\hat{h}_{m}$ are uniquely defined and are bounded measurable functions. Let $\left\{\hat{g}_{j}\right\},\left\{\hat{h}_{m j}\right\}$ be sequences of smooth functions which are uniformly bounded and which approximate $g$ and $\hat{h}_{m}$, respectively, in the $L^{1}$-norm. Consider the system (2.5)-(2.7) with $g, h$ replaced by $\hat{g}_{j}$ and $\hat{h}_{m j}$ respectively, and denote the corresponding solution by $v_{m j}$.

The $v_{m j}$ are uniformly bounded, and (by taking a subsequence) we may assume that they form a sequence which is convergent, both weakly in $L^{2}\left(\Omega_{T}\right)$ and almost everywhere in $\Omega_{T}$, to some function $v_{m}$.

Multiplying (2.5) (with $v=v_{m j}$ ) by $\phi$ ( $\phi$ as in (1.9)), performing integration by parts, and then taking $j \rightarrow \infty$, we find that

$$
\iint_{\Omega_{T}}\left[a_{m}\left(v_{m}\right) \phi_{t}+v_{m} L^{m} \phi\right] d x d t=\int_{0}^{T} \int_{\partial G} g \frac{\partial \phi}{\partial \nu} d S_{x} d t-\int_{G(0)} a_{m}\left(\hat{h}_{m}\right) \phi d x .
$$


If we take a subsequence of $\left\{v_{m}\right\}$ which is convergent to some function $v$, both weakly in $L^{2}\left(\Omega_{T}\right)$ and almost everywhere in $\Omega_{T}$, and such that also $c_{m} v_{m} \rightarrow c v$, then from the last relation we obtain the relation (1.9).

COROLlARY. If $g^{*}, h^{*}, a\left(h^{*}\right)$ are also bounded measurable functions, and if $g^{*} \geqq g, a\left(h^{*}\right) \geqq a(h)$, then $u^{*} \geqq u$ almost everywhere, where $u^{*}$ is the weak solution with data $g^{*}, a\left(h^{*}\right)$.

The proof is similar to the proof of Theorem 5. In fact, we can approximate $g^{*}, \hat{h}_{m}^{*}$ by $\hat{g}_{j}^{*}, \hat{h}_{m j}^{*}$ such that $\hat{h}_{m j}^{*}>\hat{h}_{m j}, \hat{g}_{j}^{*}>\hat{g}_{j}$ and then use the same comparison argument as in that proof.

We shall now give various other generalizations of the previous results.

(I) The results of the previous sections extend to nonlinear parabolic equations of the form

$$
d_{i}(u) \frac{\partial u_{i}}{\partial t}=\sum_{j=1}^{n} \frac{\partial}{\partial x_{j}}\left[k_{i}\left(u_{i}\right) \frac{\partial u_{i}}{\partial x_{j}}\right]
$$

In fact, one can transform the classical Stefan problem for such equations into the weak form (1.9) with a function $a(u)$ which is monotone and piecewise continuously differentiable (see [4]). We then can proceed as in the linear case.

(II) All the results of the previous sections extend with minor changes to the more general parabolic equations

$$
\alpha_{i} \frac{\partial u_{i}}{\partial t}=\sum_{j, k=1}^{n} a_{j k}(x) \frac{\partial^{2} u_{i}}{\partial x_{j} \partial x_{k}}+\sum_{j=1}^{n} b_{j}(x) \frac{\partial u_{i}}{\partial x_{j}}+c^{1}(x) u_{i} .
$$

(III) One can define a weak solution of (1.1)-(1.5) in a different manner, namely, by replacing (1.9) by

$$
\begin{aligned}
\iint_{\Omega_{T} .}\left[u L_{0}^{*} \phi+\right. & \left.\sum_{j=1}^{n} b_{j}(x) \frac{\partial u}{\partial x_{j}} \phi+a(u) \frac{\partial \phi}{\partial t}\right] d x d t \\
& =\int_{0}^{T} \int_{\partial G} g \frac{\partial \phi}{\partial \mu} d S_{x} d t-\int_{G(0)} a(h) \phi d x
\end{aligned}
$$

where $L_{0} u$ is the operator obtained from $L u$ by omitting the terms $b_{j} \partial u / \partial x_{j}$. The proof of Theorem 3 can then be modified to yield a solution to the present problem. Thus, instead of (2.5) we now take

$$
a_{m}^{\prime}(v) \frac{\partial v}{\partial t}=L^{m} v+\sum_{j=1}^{n} b_{j, m}(x, v) \frac{\partial v}{\partial x_{j}}
$$

where $b_{j, m}(x, u)$ is defined analogously to $c_{m}(x, u)$ in (2.3). An inequality of the form (2.14) can be derived. A subsequence of the $\left\{v_{m}\right\}$ is then convergent in $L^{2}\left(\Omega_{T}\right)$ to a function $v$ in $W^{1,2}\left(\Omega_{T}\right)$ for which (6.1) holds.

Thus, there exists a weak solution (in the sense of (6.1)) of (1.1)-(1.5) also in case the $b_{j}^{i}$ do not vanish identically. The proof of uniqueness in Theorem 4 , however, does not seem to extend to solutions of (6.1). 
(IV) All the results of the previous sections extend with trivial changes to the case where $\partial_{1} G, \partial_{2} G$ each consists of a finite number of hypersurfaces in $R^{n}$.

(V) All the results of the previous sections extend to the case of $m$-phase Stefan problems. In the classical formulation of this problem, there are $m-1$ different types of surfaces $S_{j}(j=1, \ldots, m-1)$. On $S_{j}$ the solution has a fixed value $\tau_{j}$, and $\tau_{i} \neq \tau_{j}$ if $i \neq j$. The generalized formulation of this problem (see [4]) still has the form (1.9), but now the function $a(u)$ has $m-1$ points of discontinuity.

(VI) Most of the results of $\$ \S 1-4$ and $\S 7$ extend to the case where the first initialboundary conditions are replaced by the second or third initial-boundary conditions. Instead of (1.2) we now have

$$
\partial u_{i} / \partial \tau_{i}+\beta_{i} u_{i}=g_{i}
$$

where $\tau_{i}$ is some external oblique derivative and $\beta_{i} \geqq 0$. One must impose suitable conditions on the $g_{i}$ or else restrict $T$ to be sufficiently small. For otherwise, the set $\Delta_{1}(t)$ (or $\left.\Delta_{2}(t)\right)$ on $G(t)$ where $v_{m}$ is strictly positive (or strictly negative) may shrink to zero. The existence proof of $\$ 2$ would then fail.

7. Asymptotic bounds for the one-phase problem. In [2] we have considered the one-phase Stefan problem and derived asymptotic bounds on the free boundary. In this section we derive, by a different method, similar bounds for the weak solution. We first state the classical problem and its generalized form and prove the existence and uniqueness of a weak solution.

Instead of (1.1)-(1.5) we have

$$
\begin{array}{cl}
\partial u / \partial t=\Delta u & \text { for } x \in G_{1}(t), t>0, \\
u=g_{1} & \text { for } x \in \partial_{1} G, t>0 \quad\left(g_{1}>0\right), \\
u=h_{1} & \text { on } G_{1}(0) \quad\left(h_{1}>0\right), \\
u=0 & \text { for } x \in \Gamma(t), t \geqq 0, \\
\nabla_{x} u \cdot \nabla_{x} \Phi=\alpha \partial \Phi / \partial t \quad \text { for } x \in \Gamma(t), t>0 .
\end{array}
$$

Here $\bigcup_{t} \Gamma(t)$ in the free boundary and $G_{1}(t)$ is bounded by $\Gamma(t)$ and $\partial_{1} G(t)=\partial_{1} G \times\{t\}$. $\partial_{1} G$ is a bounded hypersurface in $R^{n}$.

Let $(u, \Phi)$ be a solution of (7.1)-(7.5) for $t<T$. Introduce any smooth hypersurface $\partial_{2} G$ in $R^{n}$ which contains $\partial_{1} G$ in its interior, and let $v$ be the zero function defined for $0<t<T$ and $x$ in the shell bounded by $\Gamma(t)$ and $\partial_{2} G(t)=\partial_{2} G \times\{t\}$. Then $(u, v, \Phi)$ is a classical solution of a two-phase Stefan problem as in (1.1)-(1.5), except that now $h_{2} \equiv 0, g_{2} \equiv 0$.

We now define $a(u)$ as in (1.7) and then (temporarily) define a weak solution of (7.1)-(7.5) as a bounded measurable function $u$ in $\Omega_{T}$ which satisfies (1.9) with $L^{*}=\Delta$. Defining $a\left(h_{2}\right) \equiv-\alpha$, one can easily extend Theorems 1,2 to the present case.

In trying to carry out an existence proof analogous to the proof of Theorem 3, 
we encounter the need for some changes (compare Remark 1 at the end of \$2). First, we must satisfy the relation $\int_{G} a_{m}\left(v_{m}\right) \phi \rightarrow \int_{G} a(v) \phi=-\alpha \int_{G} \phi$ as $m \rightarrow \infty$. Hence we must take the $a_{m}(v)$ such that $a_{m}(0) \rightarrow-\alpha$. Note that this implies $a_{m}(v)<0$ in some interval $\left(0, \varepsilon_{m}\right)$. But then it is impossible to find a region $\Omega_{2}^{*}$ as in (2.10). Fortunately, however, it is not needed to prove (2.12) for $x \in \partial_{2} G$ (and thus one need not consider the system (2.10) for $i=2)$ since $\partial g_{2} / \partial t=0$ in (2.13).

Theorems 4-7 and 10 extend to the present case with minor changes in the proofs.

We shall assume that

$$
\begin{aligned}
\gamma\left[1+t^{(n-2) / 2}\right] & \leqq g_{1}(x, t) \leqq \gamma^{\prime}\left[1+t^{(n-2) / 2}\right] & & \text { if } n \geqq 3, \\
\gamma \log (2+t) & \leqq g_{1}(x, t) \leqq \gamma^{\prime} \log (2+t) & & \text { if } n=2, \\
\gamma & \leqq g_{1}(x, t) \leqq \gamma^{\prime} & & \text { if } n=1,
\end{aligned}
$$

where $\gamma, \gamma^{\prime}$ are positive constants. Note that if $g_{1}$ is given only for $t$ in a finite interval $[0, T]$ and if it is a positive function, then we can extend its definition to $\partial_{1} G \times[0, \infty)$ so that $(7.6)$ is satisfied.

We take $\partial_{2} G$ to be such that its interior contains a ball $|x|<\delta(T+1)^{1 / 2}$ where $\delta$ is a constant to be determined later on; it depends on $\gamma, \gamma^{\prime}$ but not on $T$. We shall prove that $u(x, t)=0$ if $|x| \geqq \delta(T+1)^{1 / 2}$. The proof will be based on Theorem 5 , which remains true for the present (one-phase) problem.

Let

$$
\hat{u}(x, t)=f\left[|x| /(t+1)^{1 / 2}\right], \quad \Phi(x, t)=|x|-\delta(t+1)^{1 / 2}
$$

where

$$
f(t)=C \int_{z}^{\infty} \zeta^{1-n} \exp \left\{-\zeta^{2} / 4\right\} d \zeta-C^{\prime} \quad\left(C, C^{\prime} \text { constants }\right)
$$

The conditions (7.4), (7.5) for $\hat{u}, \Phi$ become

$$
\begin{aligned}
2 C \delta^{1-n} \exp \left\{-\delta^{2} / 4\right\} & =\alpha \delta, \\
C \int_{\delta}^{\infty} \zeta^{1-n} \exp \left\{-\zeta^{2} / 4\right\} d \zeta-C^{\prime} & =0 .
\end{aligned}
$$

We take $\delta$ such that $\Gamma(0)$ is contained in the sphere $|x|=\delta$. From (7.9), (7.10) it follows that $C=(\alpha / 2) \delta^{n} \exp \left\{\delta^{2} / 4\right\}$ whereas $C^{\prime}$ remains bounded as $\delta \rightarrow \infty$. Hence we find that if $\delta$ is sufficiently large, then

$$
\begin{array}{ll}
\hat{u}>g_{1} & \text { on } \partial_{1} G \times[0, T), \\
\hat{u}>h_{1} & \text { on } G_{1}(0) .
\end{array}
$$

Defining $\hat{u}_{1}(x, t)=\hat{u}(x, t)$ for $x$ outside $\partial_{1} G$ and inside the ball $|x|=\delta(t+1)^{1 / 2}$ and $\hat{u}_{2}(x, t)=0$ for $x$ outside the sphere $|x|=\delta(t+1)^{1 / 2}$ and inside $\partial_{2} G$, we can apply Theorem 5 to compare $u$ with the weak solution corresponding to $\left(\hat{u}_{1}, \hat{u}_{2}, \Phi\right)$. We conclude that $u(x, t) \leqq \hat{u}_{2}(x, t)=0$ for $x$ outside $|x|=\delta(t+1)^{1 / 2}$ and inside $\partial_{2} G$. 
We can now show that the definition of a weak solution does not depend on the choice of $\partial_{2} G$ provided $\partial_{2} G$ contains the ball $|x| \leqq \delta(T+1)^{1 / 2}$ in its interior. Take, in fact, another smooth hypersurface $\partial_{2} \widetilde{G}$ which contains the ball $|x| \leqq \delta(T+1)^{1 / 2}$ in its interior, and let $\tilde{u}$ be the corresponding weak solution. We shall prove that $u=\tilde{u}$ in the set where both solutions are defined. Denote by $\Omega_{T}$ and $\tilde{\Omega}_{T}$ the cylinders where $u$ and $\tilde{u}$ respectively are defined. We may assume that $\Omega_{T} \supset \Omega_{T}$ since otherwise we can introduce a third weak solution defined in a cylinder which contains both $\Omega_{T}$ and $\tilde{\Omega}_{T}$. We now claim that $\tilde{u}$ is a weak solution also in $\Omega_{T}$. Indeed, this is easily seen by using the fact that $\tilde{u}(x, t)$ vanishes for $|x|>\delta(T+1)^{1 / 2}$. Since $u$ and $\tilde{u}$ are then both weak solutions in $\Omega_{T}$, by uniqueness, we conclude that $\tilde{u} \equiv u$ in $\Omega_{T}$.

Let $\left\{\partial_{2} G_{m}\right\}$ be a sequence of spheres $|x|=m, m>\delta(T+1)^{1 / 2}$, and denote by $u_{m}$ the weak solution when $\partial_{2} G$ is $\partial_{2} G_{m}$. By what we have proved, the $u_{m}$ define uniquely a function $u(x, t)$ for $x$ outside $\partial_{1} G$ and $0 \leqq t \leqq T$. We shall call this function the weak solution of (7.1)-(7.5) for $t<T$.

It is clear that the weak solutions defined for $t<T$ and for $t<T^{\prime}$ must coincide for $0 \leqq t \leqq \min \left(T, T^{\prime}\right)$. Thus there exists a unique weak solution for all $t>0$.

We shall now find a region where the weak solution $u$ is positive.

We assume that there exists a ball

$$
B=\{x ;|x| \leqq \beta\} \text { containing } \partial_{1} G \text { and contained in } \Gamma(0) .
$$

We first prove the following lemma.

LEMMA 3. Denote by $G_{1}(t)$ the set of points $(x, t)$ with $x$ outside $\partial_{1} G$ such that $u(x, t)>0$. Then $G_{1}(0) \times\{t\} \subset G_{1}(t)$.

Proof. Let $\left\{G_{k}\right\}$ be a sequence of domains with smooth boundary such that $\bar{G}_{k} \subset G_{k+1}, G=\bigcup_{k} G_{k}$ and such that $\partial_{1} G$ is a part of the boundary $\partial G_{k}$ of $G_{k}$. Take $k$ fixed. Then $h_{1}(x) \geqq \delta_{0}>0$ on $G_{k}$. By decreasing $\delta_{0}$ if necessary we may also assume that $g_{1}(x, t) \geqq \delta_{0}$ on $\partial_{1} G \times[0, \infty)$. Consider now the solutions $v_{m}$ of $(2.5)-(2.7)$ with suitable $a_{m}(v)$ such that $v_{m} \rightarrow v$, where $v$ is the weak solution. Recall that $v_{m} \geqq 0$. We shall compare $v_{m}$ with a function $w$ satisfying

$$
\Delta w=0 \quad \text { in } G_{k}, \quad w=\delta_{0} \quad \text { on } \partial_{1} G, \quad w=0 \quad \text { on } \partial G_{k}-\partial_{1} G .
$$

We may consider $w$ to be a solution of $a_{m}^{\prime}\left(v_{m}\right) w_{t}=\Delta w$ in $G_{k} \times(0, T)$. Since $v_{m}-w \geqq 0$ on the lateral boundary and on the base of this cylinder, the maximum principle shows that $v_{m} \geqq w$ in $G_{k} \times(0, T)$. Hence also $v \geqq w>0$ in $G_{k} \times(0, T)$. Since $k$ is arbitrary, the assertion of the lemma follows.

Using the assumption (7.11), we conclude that $u(x, t)>0$ in $G^{\prime} \times(0, T)$, where $G^{\prime}$ is the domain bounded by $\partial_{1} G$ and the sphere $|x|=\beta . u$ then satisfies the heat equation in $G^{\prime} \times(0, T)$.

Consider the function

$$
z(x, t)=\varepsilon\left\{\exp \left[-\lambda|x|^{2}\right]-\exp \left[-\lambda \beta^{2}\right]\right\}(1+t)^{(n-2) / 2} \quad(\varepsilon>0)
$$


for $n \geqq 3$. Choose $\lambda$ sufficiently large such that $\Delta z-z_{t} \geqq 0$ in $G^{\prime} \times(0, T)$. If $\varepsilon$ is sufficiently small, then $u-z \geqq 0$ on the lateral boundary and on the base of the cylinder $G^{\prime} \times(0, T)$. Applying the maximum principle to $u-z$, we conclude that $u-z \geqq 0$ throughout the cylinder. Hence, for any $0<\beta^{\prime}<\beta$, there exists $\eta>0$ such that

$$
u(x, \sigma) \geqq \eta \sigma^{(n-2) / 2} \quad \text { for any } \sigma \geqq 1, x \text { outside } \partial_{1} G,|x|<\beta^{\prime} .
$$

\section{Consider}

$$
u^{*}(x, t)=f\left(|x| / t^{1 / 2}\right), \quad \Phi^{*}(x, t)=|x|-B t^{1 / 2} \quad\left(B \sigma^{1 / 2}=\beta^{\prime}\right)
$$

for $t>\sigma$, where $f$ is the function defined by (7.8)-(7.10) with $\delta=B$. Set $u_{1}^{*}(x, t)$ $=u^{*}(x, t)$ if $|x|<B t^{1 / 2}$ and $x$ outside $\partial_{1} G$, and $u_{2}^{*}(x, t)=0$ if $|x|>B t^{1 / 2}$. Then the classical solution $\left(u_{1}^{*}, u_{2}^{*}, \Phi^{*}\right)$ of the appropriate Stefan problem for $t>\sigma$ defines a weak solution $w$. If $\sigma$ is sufficiently large, then, by (7.12), $a(u) \geqq a(w)$ on $t=\sigma$. We can now employ the corollary to Theorem 10 and thus conclude that

$$
u(x, t) \geqq f\left(|x| / t^{1 / 2}\right) \quad \text { if }|x|<B t^{1 / 2}, t>\sigma .
$$

(7.14) was proved under the assumption that $n \geqq 3$. The proof in the cases $n=1,2$ is similar.

We sum up most of the results obtained above.

THEOREM 11. Let $\partial_{1} G \in C^{2+\eta}, \eta>0$; let $g_{1}, h_{1}$ be continuous functions on $\partial_{1} G \times[0, \infty)$ and $\mathrm{Cl}\left(G_{1}(0)\right)$ respectively, coinciding on $\partial_{1} G$, and assume that $(7.6)$ holds and that $h>0$ in $G_{1}(0)$. Then there exists a unique weak solution $u$ of (7.1)-(7.5) for all $t>0 . u \equiv 0$ outside some region $|x|>\delta(t+1)^{1 / 2}$ and, if (7.11) holds, $u$ satisfies (7.14).

8. Monotonicity of $G_{1}(\sigma)$ for the one-phase problem. Throughout the next three sections we denote by $G_{1}(\sigma)$ the set on $t=\sigma$ which lies outside $\partial_{1} G \times\{\sigma\}$ and for which $a(u(x, \sigma)) \geqq 0$, and by $G_{2}(\sigma)$ the set on $t=\sigma$ which lies outside $\partial_{1} G \times\{\sigma\}$ and for which $a(u(x, \sigma))=-\alpha$. Physically, $G_{1}(\sigma)$ is the set occupied by the liquid and $G_{2}(\sigma)$ is the set occupied by the solid. We shall prove in this section the following theorem, which includes Lemma 3 as a special case.

THEOREM 12. Let $\partial_{1} G, h_{1}, g_{1}$ be as in Theorem 11. If $\sigma^{\prime}<\sigma$, then almost all the points of $G_{1}\left(\sigma^{\prime}\right)$ lie in $G_{1}(\sigma)$.

We shall express the assertion of the theorem also by saying that $G_{1}\left(\sigma^{\prime}\right) \subset G_{1}(\sigma)$ almost everywhere.

Proof. We shall need the following lemma.

LEMMA 4. Let $w$ satisfy

$$
\begin{array}{rlrl}
a(x, t) \frac{\partial w}{\partial t} & =\Delta w & & \text { in } \Omega_{T}=G \times(0, T), \\
w & \geqq 0 & & \text { on } \partial G \times(0, T), \\
w \geqq 1 & & \text { on } G,
\end{array}
$$


where $G$ is a bounded domain, $w \in C(\bar{G} \times[0, T))$ and $a(x, t)$ is a bounded measurable function satisfying $a(x, t) \geqq \gamma_{1}>0$. Then, for any $\varepsilon>0$, there exists $a \delta, 0<\delta<1$, and a subdomain $G^{*}$ of $G$ with meas. $\left(G-G^{*}\right)<\varepsilon$, such that $w(x, t)>\delta$ on $\Omega_{T}^{*}=G^{*} \times(0, T)$. $\delta$ and $G^{*}$ depend only on $\varepsilon, \gamma_{1}, G$.

Proof. Let $G^{\prime}$ be a subdomain of $G$ with smooth boundary such that meas. $\left(G-G^{\prime}\right)<\varepsilon / 2$. Let $\lambda_{0}$ be the first eigenvalue of the Laplacian $\Delta$ in $G^{\prime}$ and $\phi_{0}$ its eigenfunction, i.e. $\Delta \phi_{0}+\lambda_{0} \phi_{0}=0$ in $G^{\prime}, \phi_{0}=0$ on $\partial G^{\prime}, \phi_{0} \not \equiv 0$. As is well known, $\lambda_{0}>0$ and $\phi_{0}(x)>0$ in $G^{\prime}$. We normalize $\phi_{0}$ by $\sup _{G^{\prime}} \phi_{0}=1$.

Consider the function

$$
v_{0}(x, t)=\phi_{0}(x) e^{-\mu t} \quad \text { where } \mu=\lambda_{0} / \gamma_{1} .
$$

Then

$$
\Delta v_{0}-a \partial v_{0} / \partial t=\phi_{0} e^{-\mu t}\left(-\lambda_{0}+a \mu\right) \geqq 0 \quad \text { in } G^{\prime},
$$

and $v_{0}(x, 0)=\phi_{0}(x) \leqq 1 \leqq w(x, 0)$ on $G^{\prime}, v_{0}(x, t)=0 \leqq w(x, t)$ on $\partial G^{\prime} \times(0, T)$. By the maximum principle, $w \geqq v_{0}$ in $G^{\prime} \times(0, T)$. The assertion now easily follows.

We proceed with the proof of Theorem 12 . For simplicity we may take $\sigma^{\prime}=0$ provided instead of $u=h$ at $t=0$ we are given $a(u)=a(h)$ (i.e. $u$ is a weak solution for $0 \leqq t \leqq T$ with $a(u)=a(h)$ on $t=0, a(h) \geqq 0$ on $G_{1}(0),-\alpha \leqq a(h)<0$ outside $G_{1}(0)$, and provided $G_{1}(0)$ is any measurable set (since $G_{1}\left(\sigma^{\prime}\right)$ is a measurable set as it coincides with $\left\{\left(x, \sigma^{\prime}\right) ; x\right.$ outside $\left.\left.\partial_{1} G, a\left(u\left(x, \sigma^{\prime}\right)\right) \geqq 0\right\}\right)$.

Take a sequence $\left\{D_{k}\right\}$ of open sets, $D_{k} \supset D_{k+1}, D_{k} \supset G_{1}(0)$ such that meas. $\left(D_{k}-G_{1}(0)\right)<1 / k$, and set $D_{k}=\bigcup_{p} D_{k p}$ where each $D_{k p}$ is a domain, $D_{k p} \cap D_{k q}=\varnothing$ if $p \neq q$. We may assume that the $D_{k}$ are bounded sets lying outside $\partial_{1} G$.

Let $\varepsilon_{k}=(\alpha+1) \chi_{k}$ where $\chi_{k}$ is the characteristic function of $D_{k}-G_{1}(0)$, and let $\tilde{\varepsilon}_{k}=\tilde{\chi}_{k} / k$ where $\tilde{\chi}_{k}$ is the characteristic function of $D_{k}$.

We can approximate $a(v)$ by smooth functions $a_{m}(v)$ such that if $a(v)=a_{m}\left(v^{*}\right)$, then $v^{*} \geqq v$ and such that $a_{m}^{\prime}(v) \geqq \min \left(\alpha_{1}, \alpha_{2}\right)=1$. Note that $a_{m}(0) \leqq-\alpha$.

Define $h_{m k}$ by

$$
a_{m}\left(h_{m k}\right)=a(h)+\varepsilon_{k}(x)+\tilde{\varepsilon}_{k}(x)
$$

in $G$, where $G$ is bounded by $\partial_{1} G$ and $\partial_{2} G$ (see $\S 7$ ); we may take $G$ to contain all the sets $D_{k}$. Then

$$
\begin{array}{ll}
h_{m k}>0 & \text { in } G, \\
h_{m k}>\eta_{k} & \text { on both } G_{1}(0) \text { and on } D_{k}-G_{1}(0) .
\end{array}
$$

$\eta_{k}$ is a positive constant independent of $m$ and we can assume that $\eta_{k}<g_{1}$ on $\partial_{1} G \times[0, T)$.

For $k$ fixed and for a given $\varepsilon>0$, we take $p_{0}$ such that meas. $\left(\bigcup_{p>p_{0}} D_{k p}\right)<\varepsilon / 2^{k+1}$. 
Let $D_{k p}^{\prime}\left(p<p_{0}\right)$ be a subdomain of $D_{k p}$ with $\mathrm{Cl}\left(D_{k p}^{\prime}\right) \subset D_{k p}$ such that

$$
\sum_{p=1}^{p_{0}} \text { meas. }\left(D_{k p}-D_{k p}^{\prime}\right)<\varepsilon / 2^{k+1} \text {. }
$$

Set $D_{k}^{\prime}=\sum_{p=1}^{p_{0}} D_{k p}^{\prime}$. Then meas. $\left(D_{k}-D_{k}^{\prime}\right)<\varepsilon / 2^{k}$.

Consider the system

$$
\begin{aligned}
a_{m}^{\prime}(v) \partial v / \partial t & =\Delta v & & \text { in } G \times(0, T), \\
v & =g_{i} & & \text { on } \partial_{i} G \times(0, T) \\
v & =\zeta_{m k, j} & & \text { on } G,
\end{aligned}
$$

where $\zeta_{m k, j}$ are smooth functions satisfying

$$
\begin{array}{ll}
\zeta_{m k, j} \rightarrow h_{m k} & \text { in } L^{1}(G), \\
\zeta_{m k, j} \geqq \eta_{k} & \text { in } D_{k}^{\prime}, \\
0<\zeta_{m k, j}<H & \text { on } G,
\end{array}
$$

where $H$ is a constant independent of $m, k, j$. Such functions $\zeta_{m k, j}$ can be constructed as mollifiers of $h_{m k}$ (see, for instance [1]) provided the parameter of the mollifiers is sufficiently small (depending on $k, \varepsilon$ ).

If $g_{1}$ is sufficiently smooth and if it agrees smoothly with $\zeta_{m k, j}$ on $\partial_{1} G$, then a unique solution of (8.3) is known to exist. In the more general case where $g_{1}$ is only continuous, we can approximate it by such smooth functions $g_{1, i}$. Denoting the corresponding solution of (8.3) by $v_{m k, j i}$ and using a priori estimates, we can extract a subsequence which will converge to a solution $v_{m k, j}$ of (8.3). $v_{m k, j}$ need not be continuous on $\partial_{1} G$ (since $g$ and $\zeta_{m k, j}$ need not agree on $\partial_{1} G$ ). By the maximum principle, $v_{m k, j}>0$ in $G \times(0, T)$.

We now apply Lemma 4 to $v_{m k, j}$ in $D_{k p}^{\prime} \times(0, T)$ for any $p<p_{0}$. It follows that there exists a subdomain $D_{k p}^{\prime \prime}$ of $D_{k p}^{\prime}$ such that meas. $\left(D_{k p}^{\prime}-D_{k p}^{\prime \prime}\right)<\varepsilon / 2^{k} p_{0}$, and such that

$$
v_{m k, j}>\delta \eta_{k} \text { in } D_{k p}^{\prime \prime} \times(0, T) .
$$

Here $\delta=\delta(\varepsilon, k)$ is independent of $j, m$.

From the definition of $a_{m}(v)$ it follows that if $m$ is sufficiently large, i.e. $m \geqq m_{0}$, $m_{0}=m_{0}(k, \delta)=m_{0}[k, \varepsilon]$, then

$$
a_{m}\left(v_{m k, j}\right)>0 \text { in } D_{k p}^{\prime \prime} \times(0, T) .
$$

Now take $j \rightarrow \infty$ through such values that $v_{m k, j} \rightarrow v_{m k}$ both weakly in $L^{2}\left(\Omega_{T}\right)$ and almost everywhere in $\Omega_{T}$. From (8.6) we get

$$
a_{m}\left(v_{m k}\right) \geqq 0 \text { almost everywhere in } \bigcup_{p=1}^{p_{0}} D_{k p}^{\prime \prime} \times(0, T) \text { if } m \geqq m_{0} .
$$


The integrated form of $(8.3)$ is

$$
\iint_{\Omega_{T}}\left[a_{m}\left(v_{m k}\right) \phi_{t}+v_{m k} \Delta \phi\right] d x d t=\int_{0}^{T} \int_{\partial_{1} G} g \frac{\partial \phi}{\partial \nu} d S_{x} d t-\int_{G(0)} a_{m}\left(h_{m k}\right) \phi d x
$$

for any function $\phi$ with $D_{x} \phi, D_{x}^{2} \phi, D_{t} \phi$ continuous in $\bar{\Omega}_{T}$ and which vanishes on $G \times\{T\}$ and on $\partial G \times(0, T)$.

In order to prove (8.8), we first derive its analog for $v_{m k, j i}$, then take $i \rightarrow \infty$ and, finally, $j \rightarrow \infty$.

Now take $m \rightarrow \infty$ through such values that $v_{m k} \rightarrow v_{k}$ both weakly in $L^{2}\left(\Omega_{T}\right)$ and almost everywhere in $\Omega_{T}$. From (8.1), (8.8) we get

$$
\int_{\Omega_{T}}\left[a\left(v_{k}\right) \phi_{t}+v_{k} \Delta \phi\right] d x d t=\int_{0}^{T} \int_{\partial_{1} G} g \frac{\partial \phi}{\partial \nu} d S_{x} d t-\int_{G} a(h) \phi d x-\int_{G}\left(\varepsilon_{k}+\tilde{\varepsilon}_{k}\right) \phi d x,
$$

where $a\left(v_{k}\right)$ is the limit, almost everywhere, of $\left\{a_{m}\left(v_{m k}\right)\right\}$. Note that $a\left(v_{k}\right)$ is a measurable function and all we know about its values at points $(x, t)$ where $v_{k}(x, t)=0$ is that $-\alpha \leqq a\left(v_{k}(x, t)\right) \leqq 0$.

Take now $k \rightarrow \infty$ through such values that $v_{k} \rightarrow v$ both weakly in $L^{2}\left(\Omega_{T}\right)$ and almost everywhere in $\Omega_{T}$. From (8.9) we then obtain

$$
\iint_{\Omega_{T}}\left[a(v) \phi_{t}+v \Delta \phi\right] d x d t=\int_{0}^{T} \int_{\partial_{1} G} g \frac{\partial \phi}{\partial \nu} d S_{x} d t-\int_{G} a(h) \phi d x .
$$

Thus, $v$ is a weak solution, for $0 \leqq t \leqq T$, of the Stefan problem with $a(v)=a(h)$ on $t=0$. By uniqueness, it coincides with $u$ almost everywhere.

Now, by (8.7) it follows that $a\left(v_{k}\right) \geqq 0$ almost everywhere in $D_{k}^{*} \times(0, T)$ where $D_{k}^{*}=\bigcup_{p=1}^{p_{0}} D_{k p}^{\prime \prime}$, and meas. $\left(D_{k}-D_{k}^{*}\right)<2 \varepsilon / 2^{k}$. Hence $a\left(v_{k}\right) \geqq 0$ almost everywhere in $\left(G_{1}(0)-G_{k}^{*}\right) \times(0, T)$, where $G_{k}^{*}=\left(D_{k}-D_{k}^{*}\right) \cap G_{1}(0)$. Let $G^{*}=\bigcup_{k=1}^{\infty} G_{k}^{*}$. Then meas. $G^{*}<2 \varepsilon$ and $a\left(v_{k}\right) \geqq 0$ almost everywhere on $\left(G_{1}(0)-G^{*}\right) \times(0, T)$. It follows that also $a(u) \geqq 0$ almost everywhere on this set. Since $\varepsilon$ is arbitrary, we find that $a(u) \geqq 0$ almost everywhere on $G_{1}(0) \times(0, T)$.

Recalling that the map $t \rightarrow a(u(x, t))$ is weakly continuous from $(0, T)$ into $L^{2}(G)$, we easily see that for each $t, a(u(x, t)) \geqq 0$ for almost all $x \in G_{1}(0)$. This completes the proof of Theorem 12.

9. Strict increase of $G_{1}(\sigma)$ for the one-phase problem. Denote by $\Gamma(0)$ the outer boundary of $G_{1}(0)$. We assume in this section that $h(x) \in W^{1,2}(G)$ and that there exists a function $\Psi$ with $D_{x} \Psi, D_{x}^{2} \Psi, D_{t} \Psi$ continuous in $\bar{\Omega}_{T}$ such that $\Psi=g$ on $\partial G \times[0, T)$ and $\Psi=h$ on $V_{0} \cap G(0)$, where $V_{0}$ is a neighborhood of $\partial G$.

THEOREM 13. If $\Gamma(0)$ is of class $C^{2+\eta}(\eta>0)$, then, for any $0<t<T$, meas. $\left(G_{1}(t)-G_{1}(0)\right)>0$.

Proof. If the assertion is faise, then, in view of Theorem $12, G_{1}(t)=G_{1}(0)$ almost everywhere, for some $t=\sigma$. The same relation then holds for each $t$ with $0 \leqq t \leqq \sigma$. In the cylinder $\Omega_{*}=G_{1}(0) \times(0, \sigma)$ we have $a(u) \geqq 0$. Hence $u$ satisfies $u_{t}=\Delta u$ in the classical sense in $\Omega_{*}$. 
If $\phi \in C^{\infty}$ and has a compact support in $\Omega_{\sigma}-\bar{\Omega}_{*_{2}}$ then clearly $\iint a(u) \phi_{t} d x d t=0$. Now take $\phi \in C^{\infty}$ with compact support in $\Omega_{\sigma}$ and let $\left\{\psi_{t}(x)\right\}$ be a partition of unity for $G-\mathrm{Cl}\left(G_{1}(0)\right)$ such that the support of each $\psi_{i}$ is a compact subset of $G-\mathrm{Cl}\left(G_{1}(0)\right)$. By the previous remark $\iint a(u)\left(\psi_{t} \phi\right)_{t} d x d t=0$ for each $i$. Hence

$$
\int_{0} \int_{G-\mathrm{C}\left(G_{1}(0)\right)} a(u) \phi_{t} d x d t=0 .
$$

From the definition of a weak solution it then follows that

$$
\int_{0}^{\sigma} \int_{G_{1}(0)}\left[a(u) \phi_{t}+u \Delta \phi\right] d x d t=0 .
$$

Note that here $a(u)=u$.

Let $\Gamma_{\varepsilon}$ be the set of all points of $G_{1}(0)$ whose distance to $\Gamma(0)$ is equal to $\varepsilon$. Integrating by parts in (9.1) we find that

$$
\lim _{\varepsilon \rightarrow 0} \int_{0}^{\sigma} \int_{\Gamma_{\varepsilon}}\left(u \phi_{v}-u_{v} \phi\right) d S_{x} d t=0
$$

where. $\nu$ is the normal to $\Gamma_{\varepsilon}$.

Recall now that $\int_{G}\left|\nabla_{x} u(x, t)\right|^{2} d x \leqq C$ where $C$ is a constant independent of $t$. Hence

$$
\int_{0}^{\varepsilon_{0}}\left\{\int_{0}^{0} \int_{\Gamma_{s}}\left(u_{v}\right)^{2} d S_{x} d t\right\} d \varepsilon<\infty
$$

for some $\varepsilon_{0}>0$ sufficiently small. It follows that the inequality

$$
\int_{0}^{\sigma} \int_{\Gamma_{\varepsilon}}\left(u_{v}\right)^{2} d S_{x} d t>\frac{1}{\varepsilon}
$$

cannot hold for all $e>0$ sufficiently small. Hence

$$
\int_{0}^{\sigma} \int_{\Gamma_{\epsilon_{m}}}\left(u_{v}\right)^{2} d S_{x} d t \leqq \frac{1}{\varepsilon_{m}}
$$

where $\left\{\varepsilon_{m}\right\}$ is a sequence which decreases to zero.

It follows that for any smooth $\phi$ in $\bar{\Omega}_{\sigma}$, if $\phi=0$ on $\Gamma(0) \times(0, \sigma)$, then

$$
\begin{aligned}
\left|\int_{0}^{\sigma} \int_{\Gamma_{\varepsilon}} u_{v} \phi d S_{x} d t\right| & \leqq C_{\varepsilon} \int_{0}^{\sigma} \int_{\Gamma_{\varepsilon}}\left|u_{v}\right| d S_{x} d t \\
& \leqq C^{\prime} \varepsilon \int_{0}^{\sigma} \int_{\Gamma_{\varepsilon}}\left(u_{v}\right)^{2} d S_{x} d t \leqq C^{\prime} \varepsilon^{1 / 2} \rightarrow 0
\end{aligned}
$$

if $\varepsilon=\varepsilon_{m} \rightarrow 0$; here $C, C^{\prime}$ are constants.

From (9.2) we then infer that

$$
\int_{0}^{\sigma} \int_{\Gamma_{c_{m}}} u \phi_{\nu} d S_{x} d \sigma \rightarrow 0 \text { as } m \rightarrow \infty
$$


(9.4) was established only for smooth $\phi$ satisfying $\phi=0$ on $\Gamma(0) \times(0, \sigma)$ and with compact support in $\Omega_{\sigma}$. If $\phi(x, t)$ does not vanish in a neighborhood of $t=0$ or $t=\sigma$, then we can multiply it by a function $\zeta(t)$ vanishing in such a neighborhood. By applying (9.4) to $\zeta \phi$ and choosing appropriately a sequence of such functions $\zeta(t)$, we find that $(9.4)$ holds for $\phi$.

By truncating $\phi$ outside a neighborhood of $\Gamma(0) \times(0, \sigma)$ we also find that (9.4) holds for any $\phi$ smooth in $\bar{\Omega}_{\sigma}$ which vanishes on $\Gamma(0) \times(0, \sigma)$.

Finally, if $\phi$ is defined only in $V \times(0, \sigma]$ where $V$ is a neighborhood of $\Gamma(0)$ and if it vanishes on $\Gamma(0) \times(0, \sigma)$, then we can extend it smoothly into $\bar{\Omega}_{\sigma}$ and conclude that even for such $\phi$ the relations (9.3), (9.4) are valid.

Now let $K$ be Green's function of $u_{t}=\Delta u$ in the cylinder $G_{1}(0) \times(0, T)$ (see [1]). We represent $u(\xi, \sigma)$ in terms of the fundamental solution $K$ in the cylinder $G^{\varepsilon} \times(0, \sigma)$, where $G^{\varepsilon}$ is the domain bounded by $\partial_{1} G$ and $\Gamma_{\varepsilon}$. Applying (9.3), (9.4) to $\phi=K$, we find that

$$
u(\xi, \sigma)=\int_{G_{1}(0)} K(\xi, \sigma ; x, 0) u(x, 0) d x
$$

This shows that $u$ is a continuously differentiable function in $\mathrm{Cl}\left(G_{1}(0)\right) \times(0, \sigma]$.

Since $u \geqq 0$ in $\Omega_{*}$, the maximum principle shows that $u>0$ in $\Omega_{*}$. Since, by (9.4) (or (9.5)), $u=0$ on $\Gamma(0) \times(0, \sigma)$, we also have (see [1]) $u_{v}<0$ on $\Gamma(0) \times(0, \sigma)$. But, by $(9.2), u_{v}=0$ on $\Gamma(0) \times(0, \sigma)$, a contradiction.

The proof of Theorem 13 applies also locally. In fact one easily establishes the following result.

THEOREM 13'. Let $V$ be an open domain on $t=0$ with $V \cap G_{1}(0) \neq \varnothing$, such that $V \cap \Gamma(0) \equiv \Gamma^{\prime}(0)$ is in $C^{2+\eta}(\eta>0)$. Then for every $t, 0<t<T$,

$$
\text { meas. }\left\{[V \times\{t\}] \cap G_{1}(t)-\left[V \cap G_{1}(0)\right] \times\{t\}\right\}>0 .
$$

In Theorems 13, 13' we assume that $h(x)>0$ in $G_{1}(0)$. The proofs, however, remain true if we only assume that $h(x) \geqq 0, h(x) \not \equiv 0$ in Theorem 13 , and $h(x) \geqq 0$ in $G_{1}(0)$ and $h(x) \not \equiv$ in $V \cap G_{1}(0)$ in Theorem 13'.

10. Nonoccurrence of a "cloud" for the one-phase problem. Using Theorem 12, we shall prove in this section the following result:

ThEOREM 14. For any $0<\sigma<T$, the complement on $t=\sigma$ of $\mathrm{Cl}\left(G_{1}(\sigma)\right)$ is contained in $G_{2}(\sigma)$ almost everywhere.

Introducing the sets

$$
W=\left\{(x, t) \in \Omega_{T} ;-\alpha<a(u(x, t))<0\right\}, \quad W(\sigma)=W \cap\{t=\sigma\},
$$

we conclude that

$$
W(\sigma) \subset \mathrm{Cl}\left(G_{1}(\sigma)\right)-G_{1}(\sigma)
$$


We may consider $W$ as the weak free boundary. $W$ is determined only up to a set of measure zero. (10.1) yields:

COROllary 1. $W(\sigma)$ has no interior points on $t=\sigma$.

COROLlaRY 2. The weak free boundary $W$ has no interior points in $\Omega_{T}$.

Proof of Theorem 14. If the assertion is false, then there exists in $R^{n}$ a bounded set $S$ of positive measure such that $[S \times\{v\}] \cap \mathrm{Cl}\left(G_{1}(\sigma)\right)=\varnothing$ and such that

$$
-\alpha<a(u(x, t))<0 \text { for } x \in S .
$$

$S \times\{\sigma\}$ is contained in a bounded open set $S \times\{\sigma\}$ lying outside $G_{1}(\sigma)$. By Theorem $12,(S \times\{t\}) \cap G_{1}(t)=\varnothing$ for all $0<t<\sigma$. Hence $u=0$ in $S \times(0, \sigma)$. Taking $\phi$ smooth and with compact support in $S \times(0, \sigma)$, we get

$$
\int_{0} \int_{S} a(u) \phi_{t} d x d t=0 .
$$

Thus, the weak derivative of the function $t \rightarrow a(u(x, t))$ from $(0, \sigma)$ into $L^{2}(S)$ is zero. It follows that, for almost all $t, a(u(x, t))$ is a constant element of $L^{2}(\hat{S})$. Since the function $t \rightarrow a(u(x, t))$ is also weakly continuous, we conclude that $a(u(x, t))$ is independent of $t$ for all $t, 0<t<\sigma$. Hence

$$
a(u(x, \sigma-1 / m))=a(u(x, 1 / m)) \quad(m=1,2, \ldots)
$$

for almost all $x \in S$. Recalling the weak continuity of the map $t \rightarrow a(u(x, t))$, and taking in (10.3) $m \rightarrow \infty$ through an appropriate subsequence, we obtain

$$
a(u(x, \sigma))=a(u(x, 0)) \text { for almost all } x \in S .
$$

From (10.2), (10.4) it follows that $-\alpha<a(u(x, 0))<0$ almost everywhere on the set $S$ of positive measure, which is impossible.

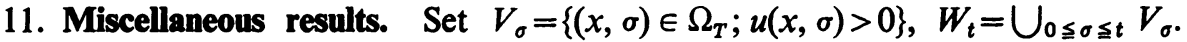
We shall make below the assumption that $u(x, t)$ is continuous in $\bar{\Omega}_{T}$. Note (by the proof of Corollary 2 to Theorem 3 ) that if $u(x, t)$ is known to be continuous in $x$, uniformly with respect to $t$, then it already follows that $u(x, t)$ is continuous in $(x, t)$.

The continuity of $u$ implies that each $V_{\sigma}$ consists of open domains. The number of these domains may increase with $\sigma$.

THEOREM 15. Let $u$ be continuous in $\bar{\Omega}_{T}$. For any $0<\sigma \leqq T$, the set $W_{\sigma}$ is connected.

Proof. We only have to prove that $W_{\sigma}$ is connected. It suffices to take an arbitrary open component $R$ of $V_{\sigma}$ and show that it can be connected in $W_{\sigma}$ to $G_{1}(0)$ (the set on $t=0$ where $h(x)>0$ ).

A continuously differentiable curve $x=x(s), t=t(s)$ along which $t^{\prime}(s) \neq 0$ will be called a monotone curve. Let $Q$ be the maximal subdomain of $W_{\sigma}$ with the property 
that each of its points can be joined to some point of $R$ by a monotone curve lying in $Q$ (except for one endpoint). Let $M=\sup _{Q} u$. We shall prove

LEMMA 5. If $\left(x^{0}, t^{0}\right)$ is a boundary point of $Q$ and $0<t^{0}<\sigma$, then $u\left(x^{0}, t^{0}\right)<M$.

Proof. If the assertion is false, then $u\left(x^{0}, t^{0}\right)=M$. Clearly $M$ is the supremum of $u\left(x, t^{0}\right)$ taken over all the boundary points $\left(x, t^{0}\right)$ of $Q$. We introduce the set $Z$ of all the boundary points $\left(x, t^{0}\right)$ of $Q$ where $u\left(x, t^{0}\right)=M . Z$ is obviously a closed set. If we prove that $Z$ is also an open set in $G \times\left\{t^{\circ}\right\}$, then $Z=G \times\left\{t^{\circ}\right\}$, which is impossible.

Let then $\left(x^{*}, t^{0}\right) \in Z$. Since $u\left(x^{*}, t^{0}\right)=M>0$, there exists an $(n+1)$-dimensional ball $K$ about $\left(x^{*}, t^{0}\right)$ such that $u>0$ in $K$. We take $K$ such that it lies in $t<\sigma$, and we denote by $K^{+}, K^{-}$the intersections of $K$ with $t \geqq t^{0}$ and $t<t^{0}$ respectively.

Employing the definition of $Q$ one finds that $K^{+} \cap Q=\varnothing$ and $K^{-} \subset Q$. By the maximum principle in $\mathrm{K}^{-}$;

$$
M=u\left(x^{*}, t^{0}\right) \leqq \max \left(\sup _{L} u, \max _{L_{0}} u\right) \leqq M
$$

where $L$ is the portion of the boundary of $K^{-}$lying in $t<t^{0}$ and $L_{0}$ is the intersection of the boundary of $K$ with $t=t^{0}$.

From (11.1) it follows that the inequalities are in fact equalities and, by the strong maximum principle, $u \equiv M$ in $K^{-}$. Thus, in particular, $K \cap\left\{t=t^{\circ}\right\}$ belongs to $Z$, and $Z$ is an open set.

LEMMA $5^{\prime}$. Lemma 5 remains true also if $t^{0}=\sigma$.

The proof is obtained by minor modifications in the proof of Lemma 5 . Thus we introduce $K^{-}$(but not $K^{+}$) and note that $K^{-} \subset Q$ and $\mathrm{Cl}\left(K^{-}\right) \cap\left\{t=t^{\circ}\right\}$ lies on the boundary $\partial Q$ of $Q$.

If we show that $\partial Q$ intersects $t=0$ at some points of $G_{1}(0)$, then $R$ can be connected in $W_{\sigma}$ to $G_{1}(0)$ and the proof of Theorem 15 is complete. Suppose then that $\partial Q$ has no points in common with $G_{1}(0)$. From Lemmas $5,5^{\prime}$ it then follows that the maximum of $u$ in $\bar{Q}$ is obtained in the set $Q \cup R$. The strong maximum principle then shows that, for some $0<\bar{\sigma} \leqq \sigma, u(x, t) \equiv M$ in (a nonempty set) $Q \cap\{t \leqq \bar{\sigma}\}$. But then the closed set $\bar{Q} \cap\{t=\bar{\sigma}\}$ (which we may assume to be nonempty) must be an open subset of $\bar{G} \times\{\bar{\sigma}\}$ (the maximality of $Q$ is hereby used). Hence $\bar{Q} \cap\{t=\bar{\sigma}\}$ $=\bar{G} \times\{\bar{\sigma}\}$, which is impossible.

REMARK 1. It is clear that Theorem 15 extends to the case of 2-phase Stefan problems. The set where $u<0$ is then also a connected set.

REMARK 2. We do not know whether the weak solution $u(x, t)$ is a continuous function when $n>1$. However, the integral

$$
\hat{u}(x, t)=\int_{0}^{t} u(x, t) d t
$$

which, by Fubini's Theorem, exists for almost all $x$ (and all $t<T$ ) is equal almost 
everywhere to a function $\hat{v}(x, t)$ which is continuous in $\Omega_{T}$. Furthermore, $\nabla_{x} \hat{v}(x, t)$ is a continuous function of $x$, uniformly with respect to $t$.

The above statement is valid also for the 2-phase Stefan problem.

To prove it, we employ the sequence $\left\{v_{m}\right\}$ which approximates $u$. We integrate the differential equation of $v_{m}$ once with respect to $t$. Introducing

$$
\hat{v}_{m}(x, t)=\int_{0}^{t} v_{m}(x, t) d t,
$$

we get $\left|\hat{v}_{m}(x, t)\right| \leqq C$ in $\Omega_{T}$, where $C$ is a constant independent of $m$. A subsequence of $\left\{\hat{v}_{m}\right\}$ converges weakly in $L^{2}\left(\Omega_{T}\right)$ and almost everywhere in $\Omega_{T}$ to a function $\hat{v}$. Employing the Sobolev inequalities for each $\hat{v}_{m}$, we find that $\hat{v}$ has the continuity properties listed above. Thus it remains to show that $\hat{v}=\hat{u}$ almost everywhere.

Now, since $v_{m} \rightarrow u$ in $L^{2}\left(\Omega_{T}\right)$, for fixed $t<T$,

$$
\int_{G} \hat{v}_{m}(x, t) \phi(x) d x \rightarrow \int_{G} \hat{u}(x, t) \phi(x) d x \quad \text { as } m \rightarrow \infty,
$$

for any bounded measurable $\phi$. By the lemma of Ascoli-Arzelà, $\hat{v}_{m}(x, t) \rightarrow \hat{v}(x, t)$ uniformly in $x$ (for a subsequence of the $m$ 's). Hence (11.2) holds with $\hat{u}$ replaced by $\hat{v}$ (and with a subsequence of the $m$ 's). It follows that, for each $t, \hat{u}(x ; t)=\hat{v}(x, t)$ for almost all $x$. Thus $\hat{u}=\hat{v}$ almost everywhere in $\Omega_{T}$.

12. Other free boundary problems. The methods of the present paper extend without difficulty to other free boundary problems. As an example, consider the problem of condensation of one drop in a supersaturated vapor of its own substance. This one-phase problem was treated in [0] in the case that the initial data are symmetric with respect to the center of the drop and the drop is spherical. If these restrictions of symmetry are removed, then the existence and uniqueness of a weak solution can be established as follows.

First consider the system for a solution $u_{R}(x, t)$ in $|x| \leqq R, 0 \leqq t \leqq T$; here $u_{R}$ is the "normalized" density of the vapor outside the drop and $u_{R}=1$ inside the drop. Existence and uniqueness of a weak solution are proved by the methods of the present section. We next take $\lim _{R \rightarrow \infty} u_{R}$ to be the weak solution for $0 \leqq t \leqq T$.

By a comparison argument one can also show that (under suitable assumptions) the set $N(t)$ where $u(x, t)=1$ contains a ball of radius $\beta(t+1)^{1 / 2}$ and is contained in another ball of radius $\delta(t+1)^{1 / 2}$. For comparison functions one can use solutions of the symmetric case whose asymptotic behavior has been studied in [0].

\section{REFERENCES}

0. A. Friedman, Free boundary problems for parabolic equations. II. Evaporation and condensation of a liquid drop, J. Math. Mech. 9 (1960), $19-66$.

1. - Partial differential equations of parabolic type, Prentice-Hall, Englewood Cliffs, N. J., 1964.

2. —, "Asymptotic behavior of solutions of parabolic differential equations and of 
integral equations" in Symposium on differential equations and dynamical systems, Academic Press, New York, 1967.

3. L. M. Graves, The theory of functions of real variables, 2nd ed., McGraw-Hill, New York, 1956.

4. S. L. Kamenomostskaja, On Stefan's problem, Mat. Sb. 53 (95) (1965), 485-514.

5. O. D. Kellogg, On the derivatives of harmonic functions on the boundary, Trans. Amer. Math. Soc. 33 (1931), 486-510.

6. O. A. Ladyzhenskaja and N. N. Uraltseva, Boundary problems for linear and quasi-linear parabolic equations. I, II, Izv. Akad. Nauk SSSR Ser. Mat. 26 (1962), 5-52, 753-780.

7. E. F. McShane, Extension of range of functions, Bull. Amer. Math. Soc. 40 (1934), 837-842.

8. L. I. Rubinstein, Two-phase Stefan problem on an interval with one-phase initial state of a heat-conducting medium, Latvijas Valsts Univ. Zinātn. Raksti 58 (1964), vyp. 2, 111-148.

9. S. L. Sobolev, Applications of functional analysis in mathematical physics, Transl. Math. Monographs, Vol. 7, Amer. Math. Soc., Providence, R. I., 1963.

10. O. A. Oleinik, On the equations of the type of nonstationary filtration, Dokl. Akad. Nauk SSSR 113 (1957), 1210-1213.

11. V. Glushko and S. G. Krěn, Fractional powers of differential operators and inclusion theorems, Dokl. Akad. Nauk SSSR 122 (1958), 963-966.

NORTHWESTERN UNIVERSITY, EVANSTON, ILLINOIS

Tel Aviv University,

Tel Aviv, Israel 